\title{
NeuroImage
}

\section{A hybrid approach to the skull stripping problem in MRI}

\author{
F. Ségonne, ${ }^{\mathrm{a}, \mathrm{b}, *}$ A.M. Dale, ${ }^{\mathrm{a}, \mathrm{b}}$ E. Busa,${ }^{\mathrm{b}}$ M. Glessner, ${ }^{\mathrm{b}}$ D. Salat, ${ }^{\mathrm{b}}$ H.K. Hahn, ${ }^{\mathrm{c}}$ and B. Fischl ${ }^{\mathrm{a}, \mathrm{b}}$ \\ ${ }^{a}$ MIT C.S.A.I. Laboratory, Cambridge, MA 02139, USA \\ ${ }^{\mathrm{b}}$ Athinoula A. Martinos Center-MGH/NMR Center, Charlestown, MA 02129, USA \\ ${ }^{\mathrm{c}}$ MeVis Bremen, Bremen, Germany
}

Received 30 September 2003; revised 15 March 2004; accepted 17 March 2004

\begin{abstract}
We present a novel skull-stripping algorithm based on a hybrid approach that combines watershed algorithms and deformable surface models. Our method takes advantage of the robustness of the former as well as the surface information available to the latter. The algorithm first localizes a single white matter voxel in a T1-weighted MRI image, and uses it to create a global minimum in the white matter before applying a watershed algorithm with a preflooding height. The watershed algorithm builds an initial estimate of the brain volume based on the three-dimensional connectivity of the white matter. This first step is robust, and performs well in the presence of intensity nonuniformities and noise, but may erode parts of the cortex that abut bright nonbrain structures such as the eye sockets, or may remove parts of the cerebellum. To correct these inaccuracies, a surface deformation process fits a smooth surface to the masked volume, allowing the incorporation of geometric constraints into the skullstripping procedure. A statistical atlas, generated from a set of accurately segmented brains, is used to validate and potentially correct the segmentation, and the MRI intensity values are locally re-estimated at the boundary of the brain. Finally, a high-resolution surface deformation is performed that accurately matches the outer boundary of the brain, resulting in a robust and automated procedure. Studies by our group and others outperform other publicly available skullstripping tools.
\end{abstract}

(C) 2004 Elsevier Inc. All rights reserved.

Keywords: Skull stripping; Brain segmentation; Watershed transformation; Template deformation; Atlas-based segmentation

\section{Introduction}

Whole-brain segmentation, called skull stripping, is an important technique for the analysis of neuroimaging data. Many applications, such as presurgical planning, cortical surface reconstruction and brain morphometry, depend on the ability to accurately segment brain from nonbrain tissue, e.g., remove extracerebral tissue such as skull, eyeballs, and skin. In addition, these techniques allow the

* Corresponding author. MIT Computer Science and Artificial Intelligence Laboratory, NE43v-614, 400 Technology Square, Cambridge, MA 02139. Fax: +1-617-258-6287.

E-mail address: florent.segonne+SkullStripping@m4x.org (F. Ségonne).

Available online on ScienceDirect (www.sciencedirect.com.) construction of detailed head models that can be used to fuse MRI data with EEG and MEG sensor information to generate spatiotemporal maps of brain activity (Dale and Sereno, 1993; Faugeras et al., 1999).

Current automatic approaches to automated skull stripping can be roughly divided into three categories: region-based, boundarybased, and hybrid approaches.

Region-based methods identify connected regions based on predefined criteria (typically intensity), employing thresholding, clustering, and morphological filtering to identify the targeted volume. While some published approaches are effective, region-based methods generally involve some degree of user interaction, and are sensitive to scanning parameters and intensity inhomogeneity. For example, Atkins and Mackiewich (1998) use thresholding and morphology techniques, combined with an anisotropic diffusion process to localize and segment the brain. Meegama et al. (2001) propose a similar approach. The method proposed by Ward (Cox, 1996; Ward, 1999) generates a segmented brain volume by assembling segmented slices. Morphological operations are used to smooth the brain envelope and refine the final segmentation. Another example can be found in Lemieux et al. (1999). Watershed techniques constitute a special case of region-based methods. The gradient intensity is usually the criterion defining connectivity, but some intensity-based approaches are used in a similar way, with the advantage of being less noise-sensitive. One of the main drawbacks of these methods is that they suffer from oversegmentation, which is the reason why they are usually followed by a postprocessing step to merge separate regions that belong to the same structure. Hahn and Peitgen (2000) proposed a solely intensity-based watershed algorithm, which makes use of a simple merging criterion to avoid the oversegmentation problem. In contrast to most region-based methods, their technique is particularly well adapted to brain segmentation, and is quite robust to intensity inhomogeneities.

- Boundary-based methods primarily rely on gradient information to locate the brain surface, usually modeled by an active contour. For instance, template-based methods incorporate shape information into the segmentation process, iteratively matching a balloon-like template to the brain surface, using image-based and smoothing forces (Dale et al., 1999; Kapur et 
al., 1995; Smith). Compared to region-based methods, these approaches seem more robust and less sensitive to image artifacts, and require less user-interaction. On the other hand, their success often depends on the quality of initialization and manual adjustment to scanning parameters. Furthermore, boundary-based segmentation produces recurrent errors in some part of the brain, such as the base of the cerebellum and temporal poles.

- Hybrid approaches combine the two previous methods. Kapur et al. (1995) propose a hybrid approach that uses morphological operations and active contour segmentation. Their method requires a preprocessing step, "Adaptative Segmentation" by Wells et al. (1995), which corrects for the gain introduced in the data by the imaging equipment. Shattuck and Leahy (in press), based on Shattuck et al. (2001), use adaptive anisotropic diffusion, edge detection and morphological erosions to identify the brain component. More recently, new hybrid approaches have been proposed to accurately locate the inner and outer surfaces of the brain, even in the depths of sulci. For this purpose, level-set methods are becoming of great use, representing the targeted evolving surface by the zero level set of a three-dimensional function. For instance, Xu et al. (1999) deform the active surface under a gradient vector field computed from a binary edge map. Motivated by the nearly constant thickness of the cortex, Zeng et al. (1999) use a coupled surface evolution to extract bounding surfaces of the cortex. Another method is proposed by Dawant et al. (1999), in which a combination of global similarity transforms and local free-form deformations to delineate internal structures of the brain. Other methods can be found in Atkins and Mackiewich (1998), Baillard et al. (2001), Fischl et al. (2002), Goldenberg et al. (2001), MacDonald et al. (2000), Pham and Prince (1999), Rajapakse (1997), Rehm et al. (1999) and Zhang et al.

Nevertheless, due to the presence of imaging artifacts, anatomical variability, varying contrast properties, and poor registration, most of these techniques do not give satisfactory results over a wide range of scan types and neuroanatomies without manual intervention. In this paper, we propose a hybrid approach to robustly and automatically segment brain from non-brain in T1-weighted MR images. We note that the algorithm does not aim at extracting the brain surface in its deepest folds; some techniques for doing so as a post-processing step can be found in Atkins and Mackiewich (1998), Dale et al. (1999), Xu et al. (1999) and Zeng (1999). Our method combines the robustness to noise that makes watershed approaches attractive, with the geometric information that are available to deformable surface algorithms. Appropriate values for the parameters of the algorithm are automatically computed during the processing. A comparison with existing techniques is reported in the final section.

\section{Methods}

Regarding brain anatomy, our approach relies on a few general assumptions:

Similar to other approaches, our first basic assumption is the connectivity of the white matter. The white matter (WM) constitutes a connected region that is bordered by gray matter (GM) and cerebrospinal fluid (CSF). In T1-weighted MR images, WM voxels have bright intensities and are surrounded by darker GM voxels and even darker CSF voxels.

- The brain surface, which separates brain from nonbrain regions, is a smooth manifold with relatively low curvature. In addition, we note that these surfaces have similar global shapes, which motivates the use of a statistical atlas to ensure that an extracted brain volume possesses the shape of a brain within a certain tolerance.

Deformable surface models are attractive techniques in that they permit the incorporation of geometric and atlas-based information into a deformation process targeting the brain surface. However, these methods often require a good initialization, positioning the initial active contour close enough to the final targeted surface to avoid local minima. On the other hand, some recent watershed methods have proven robustness to noise and could lead to a good initial extraction of the brain surface. For this reason, we have decided to combine an initial watershed brain extraction with a subsequent deformable surface model.

Thus, the skull-stripping consists of a series of sequential steps. First, some relevant parameters are estimated from the input image $I$. Next, a watershed algorithm is performed on the intensity image, with a global minimum initialized within the cerebral white matter. Finally, a deformable surface procedure is applied to the output of the watershed algorithm to recover parts of cortex that may have been erroneously removed, using smoothness constraints on the shape of the skull and atlas information. Each of these steps is discussed in detail below. Fig. 1 provides a diagram of the different steps. For simplicity, certain explanations will be carried out in Appendix A.

\section{Preprocessing}

Before applying the watershed transform on the original image, we need to compute a set of parameters required for subsequent processing. Following the work of Stephen Smith in BET (Smith), we estimate an upper bound for the intensity of the cerebrospinal fluid $\left(\mathrm{CSF}_{\mathrm{MAX}}\right)$, the coordinates of the centroid of the brain (COG),

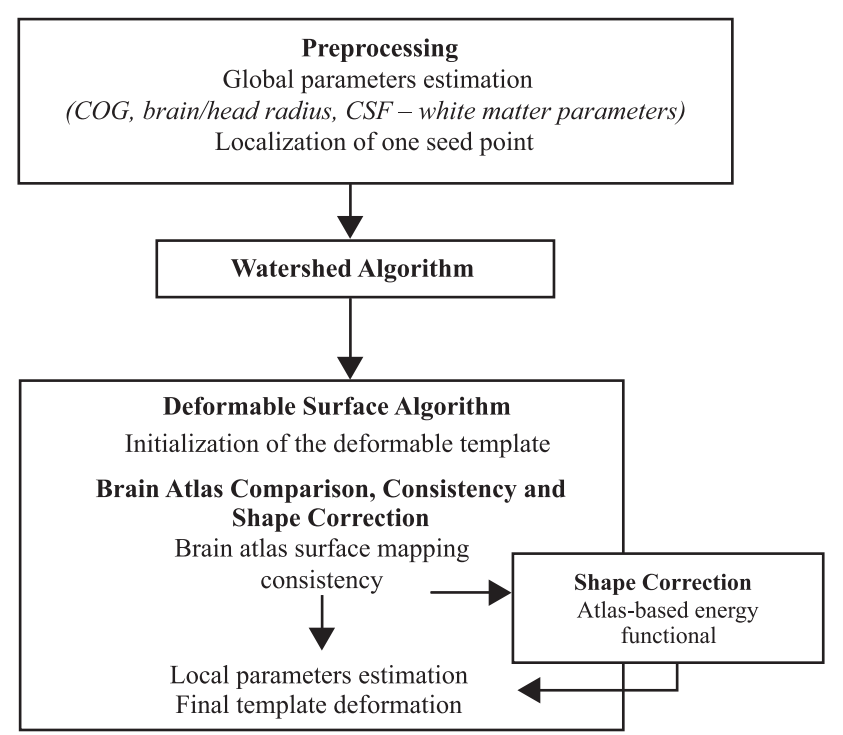

Fig. 1. The different steps of the algorithm. 
and the average brain radius (BR) (see Appendix A.1). The upper bound $\mathrm{CSF}_{\mathrm{MAX}}$ is a roughly chosen threshold, estimated to distinguish between brain and nonbrain tissue.

Next, we determine the white matter parameters. We use the fact that, in T1-weighted MRI, white matter can be identified as a uniform region localized near the interior of the brain. That is, we assume that the variance within the quasi-constant white matter intensity is small relative to other brain regions. We compute the white matter parameters from a region located at the center of the brain volume, i.e., a cubic region centered on the centroid of the brain, with an edge length of BR. Fig. 2 shows an example for the location of the cubic region of interest. This region will include nonbrain regions such as the ventricles, but voxels, with intensity smaller than $\mathrm{CSF}_{\mathrm{MAX}}$, will be ignored. Using a cumulative histogram, we compute WM estimates, which are not corrupted by presence of other tissue classes. Two bounding values for the white matter intensity values, a lower bound $\mathrm{WM}_{\mathrm{MIN}}$ and an upper bound $\mathrm{WM}_{\mathrm{MAX}}$, are computed and we estimate a value for the variance of the white matter $\sigma^{\mathrm{WM}}$ (see Appendix A.1).

Finally, we locate the white matter voxel with minimum variance in the region of interest, with intensity greater than $\mathrm{WM}_{\mathrm{MIN}}$ and smaller than $\mathrm{WM}_{\mathrm{MAX}}$. This location is then used to establish a global minimum in the image $I$, ensuring that the main basin of the watershed algorithm will represent the brain, and therefore preventing it from being merged with nonbrain regions such as the eye sockets. An example of the location of the global minimum is shown by the white cross in Fig. 2.

\section{The watershed algorithm}

The goal of the watershed algorithm is to extract an initial brain volume, removing most of the nonbrain tissue, such as scalp, skull, neck tissue... The surface of the segmented brain volume will constitute an initialization for a deformable model integrating geometrical and atlas-based information.

Watershed algorithms are based on image intensities. They typically attempt to locate the local maxima/minima of the norm of the image intensity gradient to segment the image into different connected components. The image intensity is often interpreted as height information: voxel values are used as "heights" in a landscape in which the brightest points correspond to the hills, and the darkest points represent the valleys. The image is then segmented into different basins, following the ridges of the virtual landscape. One of the main drawbacks of these techniques is that they frequently result in an oversegmentation, and appropriate merging criteria are required to postprocess the segmented image (Hahn and Peitgen, 2000). An interesting approach has been proposed by Hahn and Peitgen (2000), in which a solely intensity-based watershed algorithm is described: A simple merging criterion is defined to overcome the oversegmentation problem resulting in a fast and robust segmentation technique when relatively uniform regions are targeted.

The basic assumption of the watershed algorithm is the connectivity of the white matter. Because darker gray matter and even darker CSF surround the connected white matter, this region can be interpreted as the top of a hill in a three-dimensional virtual landscape. We apply to the MRI image a watershed transform based on the concept of preflooding to avoid oversegmentation, a common problem in watershed techniques. Our approach is exactly the one described in Hahn and Peitgen (2000). We consider the inverted gray level in the T1-weighted brain image: Under this transformation, the WM hill becomes a valley. Two points of the inverted image are connected if a path of adjacent voxels exists between them that are at least as dark as the brighter one of the two points. Under this strict definition of "connectivity", the transformation would result in an oversegmented brain. For that reason, we have to weaken our criterion for connectivity and utilize the concept of preflooding. We do so by allowing the connectivity path to contain a lower intensity than the darker of the two connected points up to a maximum difference: the preflooding height, $h_{\mathrm{pf}}$. After the watershed transform with an appropriate preflooding height, one basin should exist that represents the whole brain, and will include the previously identified white matter voxel in "Preprocessing" section. Appendix A.2 gives detailed explanations of the watershed segmentation.

The main objective of the skull-stripping algorithm is to be robust: This first step performs well in the presence of intensity nonuniformities and noise. For any given image, the parameter $h_{\mathrm{pf}}$ can be varied over a certain range without significantly changing the output. For the current algorithm, we use a value of 25 (corresponding to $25 \%$ of the maximum intensity $I_{\mathrm{MAX}}$ ). We refer to the work of Hahn and Peitgen (2000) for detailed

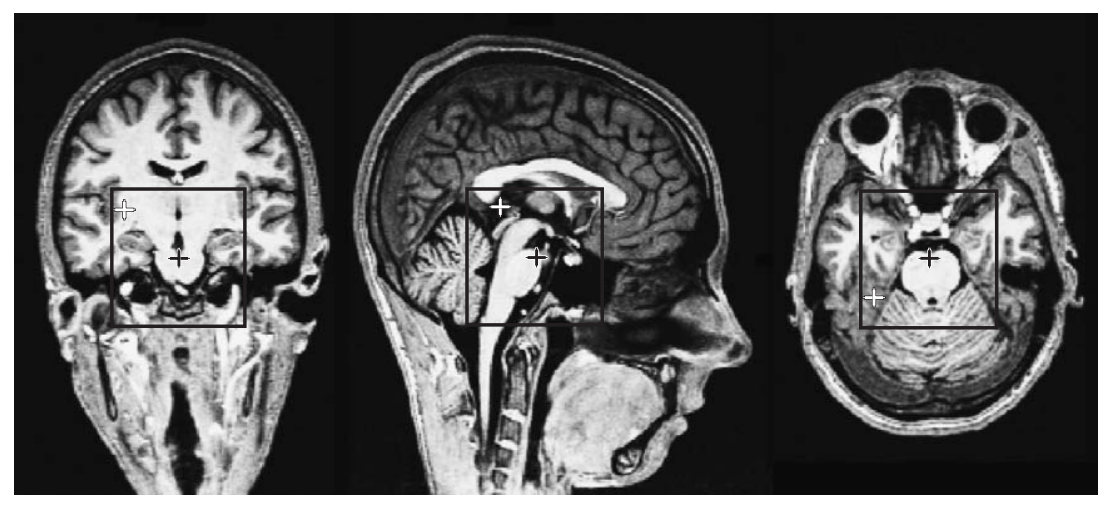

Fig. 2. The region of interest is a cube, with side length BR, centered at the COG, located with a black cross on the images. The white cross locates the position of the global minimum. 
explanations on the choice of $h_{\mathrm{pf}}$ and robustness issues. After the watershed computation, the segmented volume contains some nonbrain tissue such as CSF or some parts of the skull, and often the full brain stem. Fig. 3 shows a typical result of the watershed transform. The white arrows in Fig. 3 indicate nonbrain regions of the original image, which have been kept in the segmented brain volume. The deformable model, described in next section, incorporates geometric information such as curvature, which will be able to remove these regions. In some infrequent cases, important parts of the brain may be removed, particularly if the connectivity of the white matter is not preserved. Cerebellum white matter has frequently different intensity values from cerebral white matter due to coil sensitivity profiles or differences in intrinsic tissue properties. The complete cerebellum may not be merged into the main basin, and be detached from the whole brain by the watershed segmentation. The deformable model, integrating atlas-based shape constraints, will recover potential missing parts by correcting the shape of the template and iteratively refine the watershed segmentation by accurately fitting the surface onto the pial surface of the cortex.

\section{Deformable surface algorithm}

The watershed segmentation outputs a segmented volume, with most of nonbrain tissue removed. This brain volume is used to initialize a deformable balloon-like template. An initial template deformation is first completed using global parameters regarding the brain/nonbrain border to roughly match the boundary of the brain. Then, an atlas-based analysis verifies the correctness of the resulting surface, and modifies it if important structures have been removed. Finally, a deformation using estimates of local brain parameters will match the surface onto the true brain boundary.

We first describe the active contour model and the initialization of the template. We then describe the construction of the atlas. The final deformation integrating geometric and atlas-based information ends this section.

\section{Active contour formulation: the parametric deformable model}

To find the true brain boundary, we use the traditional framework of active contour introduced by Kass et al. (1988). In its most general form, the motion of an active contour $\mathbf{S}$ constitutes a family of surfaces $\mathbf{S}: P \times R^{+} \rightarrow R^{3},(p, t) \mapsto \mathbf{S}(p, t)$ with an evolution equation formulated as:

$\forall p \in P \quad \mathbf{S}(p, 0)=\mathbf{S}_{0}(p)$

$\forall(p, t) \in P \times R^{+} \quad \frac{\partial \mathbf{S}(p, t)}{\partial t}=\mathbf{F}(\mathbf{x}, t)$

where $\mathbf{S}_{0}$ represents the initial contour, $P$ is the parameter space and $\mathbf{F}(x, t)$ is the local force applied to the surface $\mathbf{S}$ at location $\mathbf{x}=\mathbf{S}(p, t)$. The force $\mathbf{F}$ integrates geometric, atlas-based and image information, driving the active contour toward the desired boundary.

Following the work of Dale et al. (1999), the implementation of the evolution equation is accomplished with a parametric active contour: The brain surface is modeled using a supertessellated icosahedral surface tessellation. The initial model is an icosahedron, for which each triangle was iteratively subdivided into four smaller triangles. In this common tessellation, each vertex has five or six neighbors, according to its position relative to the original icosahedron. Our deformable template uses a super-sampled icosahedron with 10,242 vertices (resulting in a mean triangle edge length of approximately $2.5 \mathrm{~mm}$ on the final brain surface).

The deformation process is driven by three different forces $\mathbf{F}=$ $\mathbf{F}_{\mathrm{S}}+\mathbf{F}_{\mathrm{MRI}}+\mathbf{F}_{\mathrm{A}}$ :

- An intrinsic curvature reducing force, enforcing a smoothness constraint on the deformed template: $\mathbf{F}_{\mathrm{S}}(\mathbf{x}, t)$. Following the work of Smith, the curvature-reducing force penalizes high local mean curvatures of the surface, ensuring smoothness of the template during the deformation process. The exact design of $\mathbf{F}_{\mathrm{S}}$ can be found in Appendix A.3.

- An MRI-based force designed to drive the template toward the true brain boundary: $\mathbf{F}_{\text {MRI }}(\mathbf{x}, t)$. In T1-weighted MRI, the contrast between gray matter and cerebrospinal fluid is large. Denoting the volume intensity at position $\mathbf{x}$ by $I(\mathbf{x})$, we define the following energy functional:

$J_{\mathrm{MRI}}=\frac{1}{2} \sum_{\text {vertices }}\left(T_{k}-I\left(\mathbf{x}_{k}^{t}\right)\right)^{2}$,

where $\mathbf{x}_{k}^{t}$ represents the coordinates of the $k$ th vertex at iteration $t$, $T_{k}$ is a local threshold at vertex $k$ that corresponds to a transition intensity between CSF and GM. The value of $I(\mathbf{x})$ is computed on

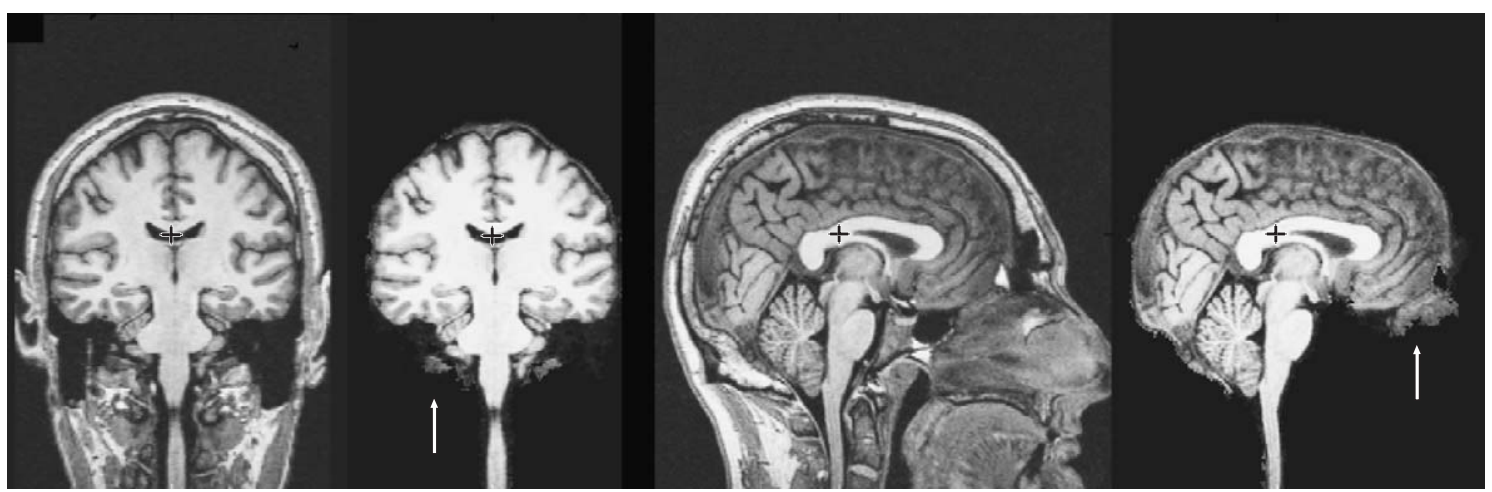

Fig. 3. Result of the watershed transform. The black cross locates the new COG. 
a subvoxel basis using trilinear interpolation. The following MRIbased force is then defined as follows:

$\boldsymbol{F}_{\mathrm{MRI}}\left(\boldsymbol{x}_{k}^{t}, t\right)=\left\{\begin{array}{l}-\boldsymbol{n}\left(\boldsymbol{x}_{k}^{t}\right) \text { if } I\left(\boldsymbol{x}_{k}^{t}\right)>\mathrm{WM}_{\mathrm{MAX}}+\sigma^{\mathrm{WM}} \\ -\frac{\partial J_{\mathrm{MRI}}}{\partial \boldsymbol{x}_{k}^{t}} \text { otherwise }\end{array}\right.$,

where $\mathbf{n}(\mathbf{x})$ denotes the unit normal vector of $S$ at location $\boldsymbol{x}$.

The first term is introduced to avoid bright regions, such as the eye sockets: in such regions, the surface is pushed inward. The second term minimizes the energy functional $J_{\text {MRI }}$, driving the surface toward local targeted threshold intensities, which locate the interface between GM and CSF.

- An atlas-based force ensures that the deformed template possesses the shape of a brain within certain tolerance: $\mathbf{F}_{\mathrm{A}}(\mathbf{x}, t)$. The force $\mathbf{F}_{\mathrm{A}}$ is defined in the next section.

Taking a forward-difference approach to discretizing the evolution equation, we update the coordinate $x_{k}^{t}$, of each vertex $k$ at iteration $t^{\prime}=t+1$ according to the forces mentioned above:

$\boldsymbol{x}_{k}^{t+1}=\boldsymbol{x}_{k}^{t}+\left[\boldsymbol{F}_{\mathrm{S}}\left(\boldsymbol{x}_{k}^{t}, t\right)+\boldsymbol{F}_{\mathrm{M}}\left(\boldsymbol{x}_{k}^{t}, t\right)+\boldsymbol{F}_{\mathrm{A}}\left(\boldsymbol{x}_{k}^{t}, t\right)\right] \delta t$.

We use a forward time step of 0.5 .

\section{Initialization of the deformable model}

Before alignment of the deformable model, no shape information is available and the atlas-based force $\mathbf{F}_{\mathrm{A}}$ is set to zero. The template is initialized as follows.

We first center the template at recalculated COG coordinates (see Fig. 3), with its radius set to include the whole previously segmented brain. Once the initial spherical template has been positioned, we gradually deform it through a series of iterative steps, using the segmented brain produced by the watershed process as a mask. In this stage, the MRI-based force $\mathbf{F}_{\text {MRI }}$ is simple: The force acting on each vertex is designed to drive the surface to regions out of the segmented brain (i.e., with $0 \mathrm{MRI}$ intensity values), repelling the surface outward from contiguous regions consistent with the segmented brain (i.e., with strictly positive MRI intensities). The yellow surface in Fig. 4 illustrates the results of this coarse initialization. The balloon-like template has been deformed onto the segmented volume.

Next, we estimate a set of global parameters including the mean intensity and variance of CSF and gray matter intensities, denoted by $\mu^{\mathrm{CSF}}, \mu^{\mathrm{GM}}, \sigma^{\mathrm{CSF}}$, and $\sigma^{\mathrm{GM}}$, respectively. These parameters will be used to refine the coarse initialization. Our main assumption is that the contrast between gray matter and CSF is sufficiently large that using unique predefined global transition intensity should suffice to distinguish the two tissues. We compute these parameters by examining the intensity values along the surface normals, extending from a few millimeters inward to a few millimeters outward from the previously calculated surface (see Appendix A.3). Assuming normal distributions for the CSF and the GM, we compute a global threshold based on the Mahalanobis distance:

$T=\left(\mu^{\mathrm{CSF}} \times \sigma^{\mathrm{GM}}+\mu^{\mathrm{GM}} \times \sigma^{\mathrm{CSF}}\right) /\left(\sigma^{\mathrm{CSF}}+\sigma^{\mathrm{GM}}\right)$,

to distinguish between brain and nonbrain tissue.

Finally, we use this global parameter $T$ to refine the coarse initialization. Each vertex of the tessellation is moved toward the desired threshold intensity according to the previously defined MRI-based force, $\mathbf{F}_{\text {MRI }}$, in "Active contour formulation: the parametric deformable model" section, where each local threshold $T_{k}$ is set to the constant global threshold $T$. Some regions of the brain are difficult to distinguish from nonbrain regions, such as the temporal lobes or the base of the cerebellum; in these complex areas, where the boundary of the brain is not easy to find, a general MRI-based force could occasionally expand the surface away from the correct boundary into nonbrain tissues. In addition, the use of a global threshold is not sufficient in the presence of strong image inhomogeneities. Therefore, we stop the iterative deformation of the template after 40 iterations, preventing large deviations from the expected final surface. The resulting surface should approximately follow the brain/nonbrain boundary. Fig. 4 illustrates the results of this process.

Brain atlas comparison, consistency and brain shape correction

As we pointed out in the last section, the watershed computation may rarely cause oversegmentation, and remove some brain voxels. In our experience, this kind of oversegmentation often leads to the removal of much or all of the cerebellum. To detect and correct these errors, which are mainly due to a deterioration of the apparent connectivity of the white matter, the surface is compared to an atlas containing geometric information compiled from successfully segmented brains. The atlas integrates two types of information, which effectively models the shape of a brain: The distance to the center of gravity, which contains important information regarding the location of the brain surface in comparison with a statistical average shape, and the curvature, which will model the convexity of the surface. In comparison with accurate brain segmentations, incorrectly removed regions often lead to brain surface patches with lower distances to COG and different convexities. On the other hand, erroneously included regions frequently produce surface patches with higher distances to $\mathrm{COG}$.

Based on its local curvature map and its distance to the center of gravity map, which we first smooth and normalize over space (zero mean and unit variance), the surface correctness is evaluated and the errors precisely localized. By normalizing the fields locally attached to the surface, we avoid incorporating prior information on the expected size of the brain, making the algorithm more general. If necessary, based on the information given by the brain atlas, the shape of the surface is iteratively corrected, as detailed below.

We will make use of the following notation: the curvature at vertex $k$ is $\operatorname{curv}_{k}$ and the distance from the $k$ th vertex to COG is $d_{k}^{\mathrm{COG}}=\left\|\mathbf{x}_{k}^{t}-\mathbf{x}_{\mathrm{COG}}\right\|$. We denote $\mathbf{X}_{k}$ as the two-dimensional vector formed by the two fields: $\mathbf{X}_{k}=\left(\operatorname{curv}_{k}, d_{k}^{\mathrm{COG}}\right)^{T}$.

Mapping the brain surface onto a sphere. We base our consistency assessment on two fields that include much of the requisite information: the mean curvature of the surface and the distance to its COG. Before calculating these two fields, we first smooth the surface by a series of iterative steps. The mean curvature map is a measure of the convexity of the surface, and the distance to the COG contains information concerning the corrective deformation field (between the surface and the atlas). To assess the validity of the segmentation, we need to map the surface into a parameterizable shape, which will allow us to compare surfaces across subjects. The sphere is a natural choice as it allows the preservation of the topological structure of the original surface (no cuts 
need to be introduced preserving the local connectivity), and it retains much of the computational attractiveness of a flat space (which would not be the case with asymmetric surfaces such as ellipsoids). We note that some high-resolution cortical coordinate systems based on this shape have been proposed in the literature
(Fischl et al., 1999a,b; Thompson and Toga, 1996; Thompson et al., 1996; Van Essen and Drury, 1997). In our case, the spherical surface will facilitate the rigid registration of the individual surface with an atlas, and will allow us to calculate the maximum likelihood at each iteration of the template deformation in a

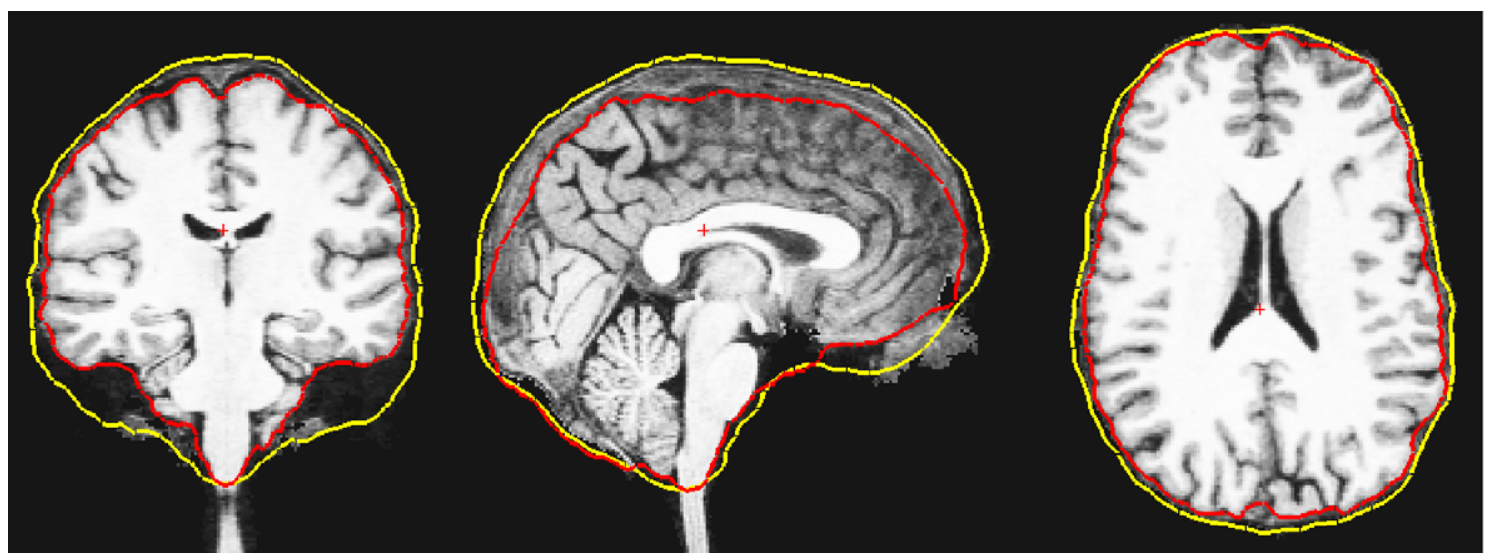

Fig. 4. Initialization of the deformable template. The yellow surface represents the initial template matched onto the segmented volume used as a mask. The red curve is the result of the matching with global parameters.

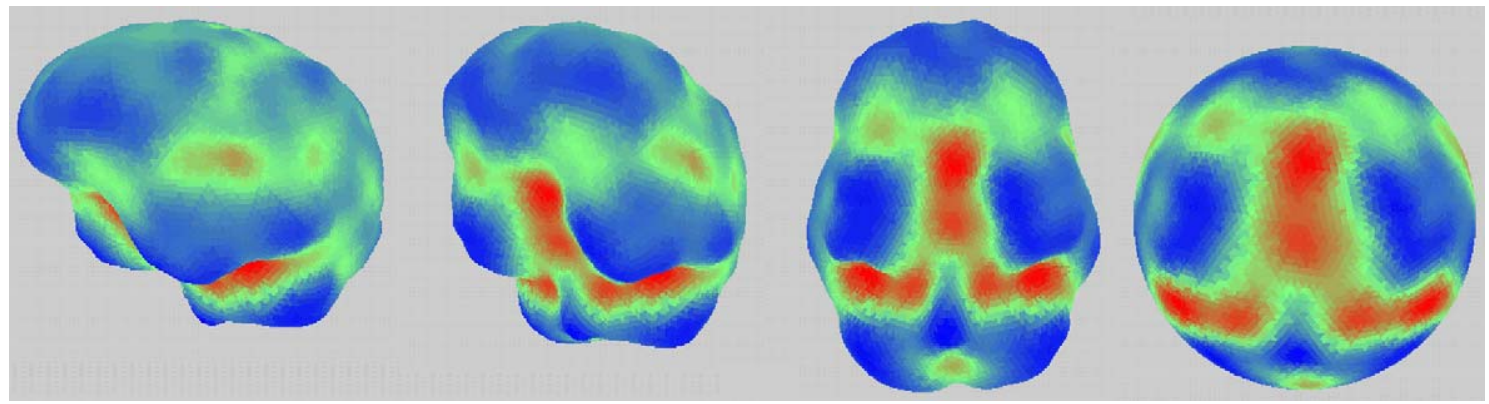

Fig. 5. Three views of the curvature field and its mapping onto a sphere.

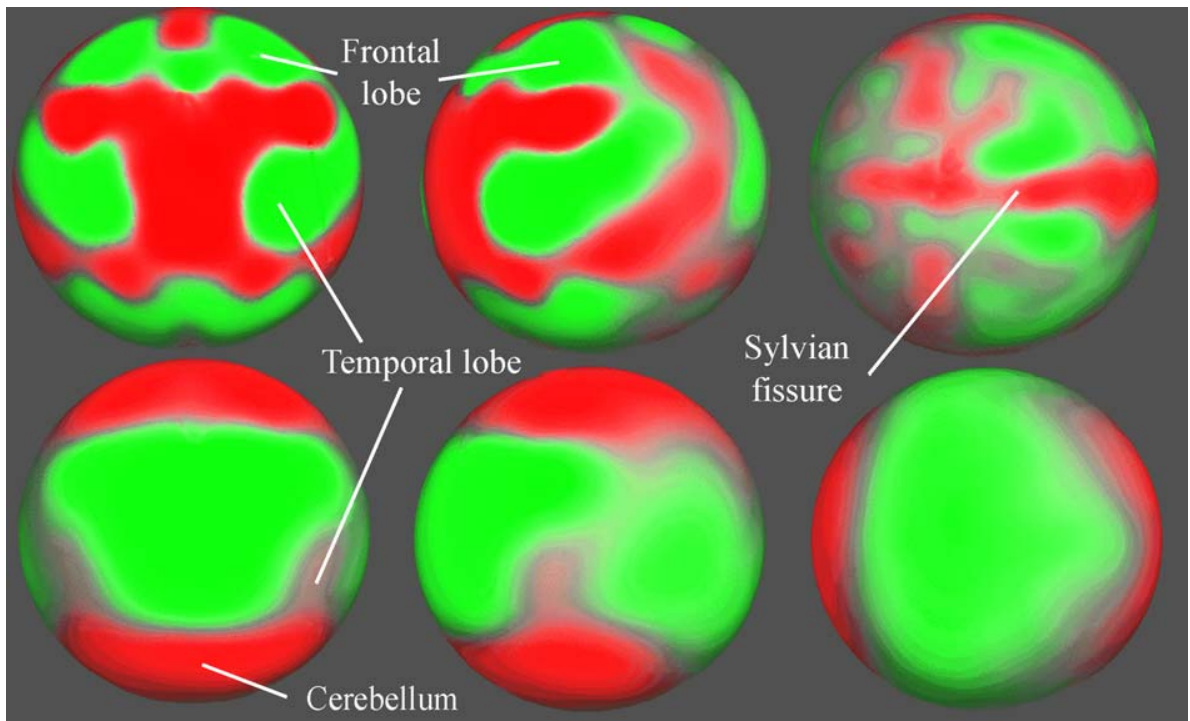

Fig. 6. Ventral, lateral, and dorsal views of the curvature field (first row) and distance to COG field (second row). 
simple manner. Therefore, we need to find a mapping from the brain surface onto a sphere. Fortunately, we can avoid unfolding our surface (Dale et al., 1999; Fischl et al., 1999b, 2001), by noting that the initial tessellated surface is modeled using an icosahedron, meaning that our original surface is exactly a tessellated sphere (see Fig. 5).

Atlas construction. The first step in verifying the shape of a surface is the construction of an atlas that contains the information necessary to detect and correct inaccuracies in the segmentation. We build this atlas from a training set of accurately segmented brains for which the curvature and distance to COG fields are computed, smoothed (1-cm Gaussian smoothing) and normalized (zero mean and unit variance). We assume that the curvature and distance fields follow two nonstationary uncorrelated Gaussian distributions in the spherical atlas space. Future work will incorporate the full covariance matrix.

First, a nonaligned template is built, containing the mean and variance of each field at each vertex. Next, each brain is rigidly aligned with the template and a new canonical surface is constructed; we note that the two fields are used to align each brain with the canonical template. Details of the alignment procedure are given in the next sections. Fig. 6 shows three different views of the spherical atlas, with the means of the curvature and distance fields.

Consistency of the shape of the surface. Once an initial representation of the brain surface has been found (see "Initialization of the deformable model" section), the curvature and COG distance fields are computed and spatially smoothed with a surface-based Gaussian filter (1-cm Gaussian smoothing). Next, the mapped sphere is rigidly aligned with the canonical surface. Assuming Gaussian distributions for the curvature and distance fields, we compute the maximum-likelihood estimate of the alignment $R^{*}$, by minimizing the following energy functional:

$J_{\mathrm{R}}=\frac{1}{2} \sum_{\text {vertices } k}\left(\boldsymbol{X}_{k}-\overline{\boldsymbol{X}}_{k}\right)^{T} \cdot \overline{\boldsymbol{V}}_{k}^{-1} \cdot\left(\boldsymbol{X}_{k}-\overline{\boldsymbol{X}}_{k}\right)$,

where $\mathbf{X}_{k}$ is a two-dimensional vector containing the curvature and distance fields at the $k$ th vertex, $\overline{\mathbf{X}}_{k}=\overline{\mathbf{X}}\left(\phi^{R}(k), \theta^{R}(k)\right)$ is a mean vector obtained from the parameterized template, and $\overline{\mathbf{V}}_{k}$ $=\overline{\mathbf{V}}\left(\phi^{R}(k), \theta^{R}(k)\right)$ the corresponding covariance matrix. $\phi^{R}(k)$ and $\theta^{R}(k)$ are the spherical $(\phi, \theta)$ coordinates of the $k$ th vertex after rotation by an alignment transform $R$. As previously stated, we assume that the two fields are uncorrelated and hence $\overline{\mathbf{V}}_{k}$ is diagonal.

After alignment, the energy functional $J_{R^{*}}$ provides a measure of the alignment error. An incorrect segmentation will lead to regions with large errors, as the result of aligning dissimilar regions. A vertex is said to be inaccurate if it satisfies $\left(\mathbf{X}_{k}-\overline{\mathbf{X}}_{k}^{*}\right)^{T} \cdot \overline{\mathbf{V}}_{k}^{*-1} \cdot\left(\mathbf{X}_{k}-\overline{\mathbf{X}}_{k}^{*}\right)>(2.6)^{2}$. Assuming Gaussian distribution, this means that the likelihood of a vertex to be classified as inaccurate is less than $1 \%$. We first localize the incorrect vertices, and then identify regions with a simple erosion-dilatation step. The overall segmentation is then said to be invalid if the number of incorrect vertices is greater than $1 \%$. Fig. 7 shows the resulting error map for a case in which the segmentation fails and removes a large portion of the cerebellum. In this case, the number of inaccurate vertices was about $10 \%$.

Correction of the geometry of the surface. If the surface segmentation fails to give the expected contour, we correct its shape with a series of deformations that iteratively minimize an energy functional $J_{\mathrm{G}}$. The mean vectors $\overline{\mathbf{X}}_{k}$ and covariance matrices $\overline{\mathbf{V}}_{k}$ of the parameterized template, which are zero mean and unit variance over the whole spherical space, are scaled such that they match the means and variances of the fields of the incorrect surface, ignoring inaccurate vertices.

Ultimately, the energy that we would like to minimize would be the following:

$J_{\mathrm{G}}=\frac{1}{2} \sum_{\text {vertices } k}\left(\mathbf{X}_{k}-\overline{\mathbf{X}}_{k}^{*}\right)^{T} \cdot \overline{\boldsymbol{V}}_{k}^{*-1} \cdot\left(\boldsymbol{X}_{k}-\overline{\boldsymbol{X}}_{k}^{*}\right)$,

where $\overline{\mathbf{X}}_{k}^{*}$ and $\overline{\mathbf{V}}_{k}^{*}$ are the previous projected and scaled mean vectors and covariance matrixes. We note that $\boldsymbol{X}_{k}$ depends on a set of neighbors of the vertex $k$, necessary to calculate the local curvature at $k^{\text {th }}$ vertex. The resulting gradient is quite complex, due to the curvature term that leads to a gradient that is dependent on the square of the inverse of the vertex spacing, and is therefore somewhat numerically unstable. Therefore, we have adopted a different approach to minimize the energy.

In the regions labeled as incorrectly segmented, we correct the shape of the tessellated surface according to the following atlas-base force:

$\mathbf{F}_{\mathrm{A}}\left(\mathbf{x}_{k}^{t}, t\right)=\frac{1}{2} \tanh \left(\left\|d_{k}^{\mathrm{COG}}\right\|_{k}^{M}\right) \cdot \mathbf{n}\left(\mathbf{x}_{k}^{t}\right)$

with $\left\|d_{k}^{\mathrm{COG}}\right\|_{k}^{M}$ being the signed Mahalanobis distance for the distance field at the $k^{\mathrm{th}}$ vertex: $\left\|d_{k}^{\mathrm{COG}}\right\|_{k}^{M}=\left(d_{k}^{\mathrm{COG}}-\bar{d}_{k}^{\mathrm{COG}}\right) / \bar{\sigma}_{k}^{\mathrm{COG}}$. The force $\mathbf{F}_{\mathrm{A}}$ pushes the surface toward its expected shape, minimizing the functional $J_{\mathrm{G}}$. A small number of iterative steps is usually enough to correct the surface. Fig. 8 illustrates this process, with a deformation, which recovers the cerebellar hemisphere.

\section{Local parameters estimation and final deformation}

Once the shape has been corrected, the resulting template closely follows the pial surface of the brain. However, intensity inhomogeneities can significantly degrade the performance of methods that assume that the intensity value of a tissue class is constant over space. This could be particularly problematic in the cerebellum, where the tissue intensity is often different from the intensity of the cerebral cortex, due to differences in intrinsic tissue properties (e.g., T1) and coil sensitivity profile. To avoid this, we compute local intensity values. The border of the brain is localized along the surface normal, as the region, where the directional 'inward' gradient reaches its maximum and for which the immediately exterior intensity value is close to the previously estimated CSF intensity. The local CSF and GM intensities are computed from the voxels located immediately outside and inside the border position. From these local values, we compute the second-neighboring mean and variance values for each tissue (CSF and gray matter: $\mu_{k}^{\mathrm{CSF}}, \mu_{k}^{\mathrm{GM}}, \sigma_{k}^{\mathrm{CSF}}$, and $\left.\sigma_{k}^{\mathrm{GM}}\right)$, and we set, for each vertex, a transition threshold $T_{k}$. Assuming local normal distributions, the threshold is based on the point at which the Mahalanobis distances are equal:

$T_{k}=\left(\mu_{k}^{\mathrm{CSF}} \times \sigma_{k}^{\mathrm{GM}}+\mu_{k}^{\mathrm{GM}} \times \sigma_{k}^{\mathrm{CSF}}\right) /\left(\sigma_{k}^{\mathrm{CSF}}+\sigma_{k}^{\mathrm{GM}}\right)$

The final deformation process is driven by the previously defined 
curvature-reducing and MRI-based forces, $\mathbf{F}_{\mathrm{S}}$ and $\mathbf{F}_{\mathrm{MRI}}$, and the following atlas-based force:

$\boldsymbol{F}_{\mathrm{A}}=\boldsymbol{F}_{\mathrm{D}}+\boldsymbol{F}_{\mathrm{C}}$, with

$\boldsymbol{F}_{\mathrm{D}}=\lambda_{\mathrm{D}} \frac{\left(d_{k}^{\mathrm{COG}}-\bar{d}_{k}^{\mathrm{COG}}\right)}{\bar{\sigma}_{k}^{\mathrm{COG}^{2}}} \cdot \boldsymbol{n}\left(\boldsymbol{x}_{k}^{t}\right)$, and

$\boldsymbol{F}_{\mathrm{C}}=\lambda_{\mathrm{C}} \frac{\left(\operatorname{curv}_{k}-\overline{\operatorname{curv}}_{k}\right)}{\bar{\sigma}_{k}^{\operatorname{curv}^{2}}} \cdot \boldsymbol{n}\left(\boldsymbol{x}_{k}^{t}\right)$

The update force $\mathbf{F}_{\mathrm{D}}$ is derived from the functional $J_{\mathrm{G}}$ when only the 'distance to COG' field is considered; assuming Gaussian distributions, it corresponds to a derivative of the maximumlikelihood estimate. The second term $\mathbf{F}_{C}$ tends to push the surface i - nward when the local curvature is greater than the template, and to push outward if it is smaller. The two constants $\lambda_{\mathrm{D}}$ and $\lambda_{\mathrm{C}}$ are empirically set to 0.25 and 0.025 , respectively. Convergence is achieved when the maximum displacement falls below a certain threshold of $0.5 \mathrm{~mm}$. The set of all necessary parameters is summarized in Appendix A.4. The final surface accurately matches the pial surface of the brain (see Fig. 9).

\section{Assessment of the results and discussion}

Stripping the skull and other nonbrain tissues from the structural images of the head is a challenging and critical component for a variety of post-processing tasks. Large anatomical variability among brains, different acquisition methods, and the presence of

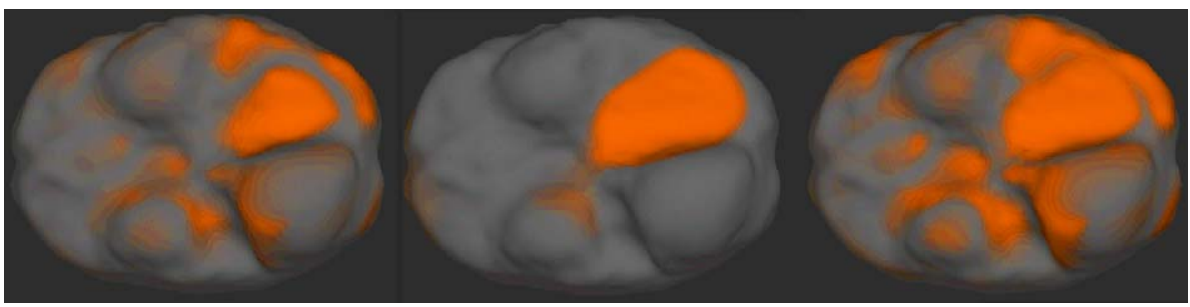

Fig. 7. The curvature, distance and global error maps

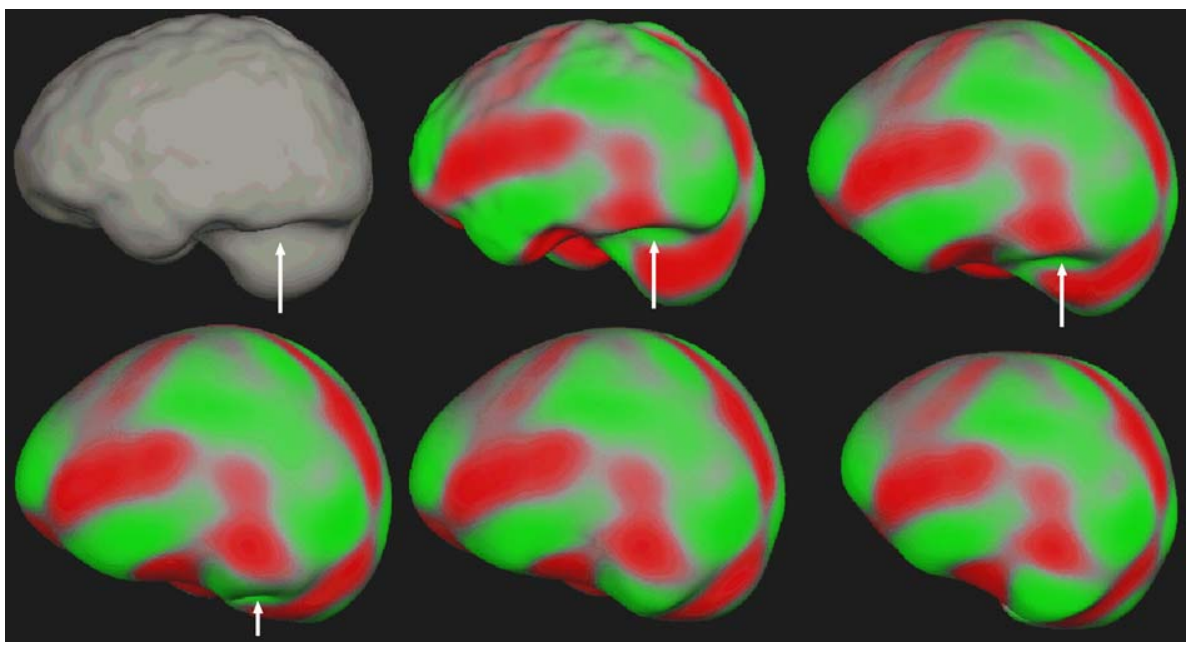

Fig. 8. The corrective deformation process with the curvature field of the canonical template projected for visualization. The white arrows locate the missing part of the cerebellum that is iteratively recovered.

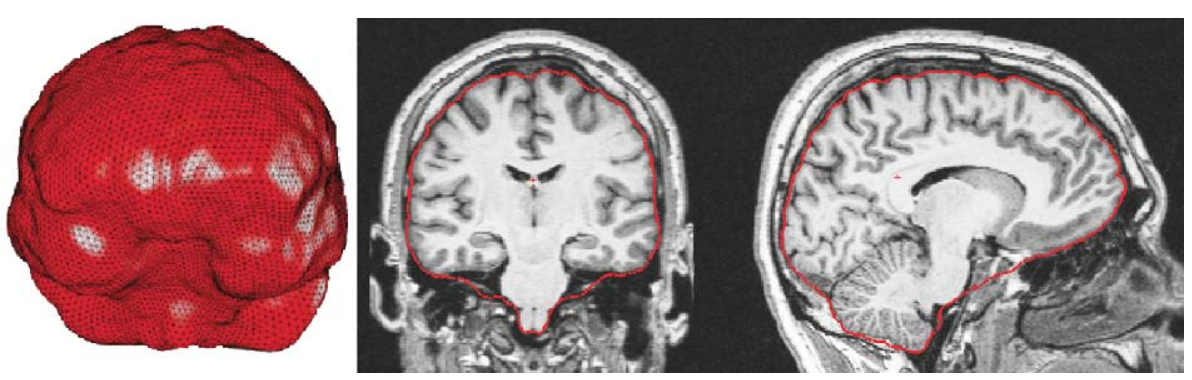

Fig. 9. A final supersampled surface. 
artifacts increase the difficulty of designing a robust algorithm, thus current techniques are often susceptible to problems and require manual intervention. To validate the proposed algorithm, we compared it to four existing techniques, and suggest ways of characterizing the correctness of the segmentation.

\section{The validation problem}

We compared our algorithm to four other existing automated skull-stripping programs: the FMRIB's Brain Extraction Tool (BET v1.2: Smith), Freesurfer's strip skull (denoted FSS; Dale et al., 1999), Hahn and Peitgen's (2000) watershed algorithm (denoted WAT), and Brain Surface Extractor (BSE in Shattuck and Leahy, in press; Shattuck et al., 2001). We will refer to our method as HWA. To assess the validity of each segmentation, we ran a study on $43 \mathrm{~T} 1$ weighted brain volumes that had also been manually skull stripped. Hence, we were able to use the manually segmented brain images as a gold standard for comparison.

\section{Presentation of different algorithms}

The five selected skull-stripping programs are all rapid. HWA is the slowest method with an average time of $5 \mathrm{~min}$ (on a $1 \mathrm{GHz}$ Pentium III running Linux), due to the spherical registration. FSS assumes that the white matter intensity distribution peaks around a specific value, therefore requiring preprocessing (Dale et al., 1999). Other methods do not require any preprocessing. We briefly present the main concepts of each method.

WAT: Hahn and Peitgen's skull stripping corresponds to a watershed segmentation as the one described in "The watershed algorithm" section and in Hahn and Peitgen (2000).

- BSE: Brain Surface Extractor-version 2.99—combines edge detection and morphology-based techniques. Adaptive anisotropic diffusion, edge detection and morphological erosions are used to identify the brain component (based on Shattuck et al., 2001).

The three other methods use deformable surfaces with different driving forces.

- FSS: The Dale et al. algorithm uses a linear smoothness constraint for the curvature reducing force and an intensitybased force described in Dale et al. (1999).

- BET: Brain Extraction Tool-version 1.2-makes use of a nonlinear smoothness constraint, as the one discussed in Appendix A.3, and of an intensity-based force described in Smith. The MRI-based force of BET's algorithm is based on regional properties of the image and the thresholds pushing the template outward are locally computed at each vertex.

- HWA: Our hybrid approach makes use of local statistics (see "Local parameters estimation and final deformation" section) for the template deformation, and integrates an atlas-based term constraining the shape of the brain. The template is initialized by a watershed presegmentation.

Description of the validation data

Validation data consisted of two data sets. All scans were MP-RAGES.

- First data set: Seventeen scans were acquired in the past 2 years using a Siemens Sonata system with the following parameters:
TR: $7.25 \mathrm{~ms}$; TE: $3.22 \mathrm{~ms}$; TI: $600.00 \mathrm{~ms}$; flip angle: $7.00^{\circ}$; $1.3-\mathrm{mm}$ sections (resampled to $1 \mathrm{~mm}$ isotropic). This data set consists of 8 young (YNC), 7 elderly normal controls (ENC), and 2 Alzeihmer's (AD).

- Second data set: A second data set with 26 scans was acquired using a Siemens Vision system in 1994/1995 with the following parameters: TR: $9.70 \mathrm{~ms}$; TE: $4.00 \mathrm{~ms}$; TI: 621.00 $\mathrm{ms}$; flip angle: $10.00^{\circ} ; 1.25$ sections (resampled to $1-\mathrm{mm}$ isotropic). Data comes from studies reported in Buckner et al. (2000) and Logan et al. (2002) and also later subjects imaged using the same anatomic protocol. ${ }^{1}$ This data set consists of 6 YNC, 14 nondemented and 6 demented adults.

\section{Risk evaluation}

To compare the performance of various segmentation techniques, we compute different coefficients reflecting how well two segmented volumes match. The manually segmented brains are used as a gold standard, and the automatically extracted brains are compared to them. Some of the automated algorithms include the third and fourth ventricles or the full brain stem in the final segmentation, while some other techniques remove these structures. To provide fair comparison between methods, the third and fourth ventricles and the brain stem were excluded from manual and automated segmentations.

Jaccard similarity coefficient. The Jaccard similarity coefficient $J$ is formulated as $J=\operatorname{vol}(A \cap B) / \operatorname{vol}(A \cup B)$, where $A$ is the brain region of the manually stripped image, $B$ is the automatically skull stripped region, and $\operatorname{vol}(X)$ denotes the volume of the region $X$. A Jaccard similarity coefficient of 1.0 represents perfect overlap, whereas an index of 0.0 represents no overlap. The Jaccard similarity coefficient is related to the Dice coefficient $D=2 \operatorname{vol}(A \cap B) / \operatorname{vol}(A+B)=2 J /(1+J)$.

False-positives and false-negatives. We define the probability of a miss $p_{\mathrm{m}}$ by the number of false negatives over the total volume of $A \cup B$. The probability of a miss is given by $p_{m}=$ $\operatorname{vol}(A \backslash B) / \operatorname{vol}(A \cup B)$, where $A$ represents the gold standard segmentation and $B$ an automated segmentation. Similarly, the probability of false detection $p_{\mathrm{f}}$ is related to the number of false positives by $p_{\mathrm{f}}=\operatorname{vol}(B \backslash A) / \operatorname{vol}(A \cup B)$. The Jaccard similarity coefficient is directly related to the false detection probability and the probability of a miss through the formula: $J=1-p_{\mathrm{m}}-p_{\mathrm{f}}$

Risk evaluation. In our view, it is significantly more important to avoid removing any brain structures than to remove all nonbrain structures. Skull-stripping methods are often used as a preprocessing step in cortical surface reconstruction (Dale et al., 1999; Fischl and Dale, 2000; Fischl et al., 1999a, 2001), or other morphometric analysis. Although skull-stripping techniques generate masks that can be used on the original data and therefore do not lead to lost information, postprocessing algorithms do not try to regain information from the exterior of the skull-stripped volume. For that reason, we define the following error function: $E(c)=\left(p_{f}+c p_{\mathrm{m}}\right) /(1+c)$. A factor of $c>1$ penalizes the eliminated brain voxels more than the added

\footnotetext{
${ }^{1}$ We thank Randy Buckner and the Washington University Alzheimer's Disease Research Center for providing the second data set.
} 
nonbrain voxels, implying that we would equate retaining $c$ nonbrain voxels with removing 1 brain voxel.

\section{Results and discussion}

The results of the study are reported in Tables 1 and 2 and Figs. 10-12. Our method performed the best overall. In most cases, our algorithm was able to precisely determine the pial boundary of the brain, resulting in an accurate segmentation. Also, our method performed well independently of the data set, and proved to be more robust than others.

\section{First data set}

HWA performed the best followed by BSE and FSS. In one case, BSE identified the wrong component and completely removed the brain volume, leading to a Jaccard similarity coefficient of 0 . We did not include this subject in the BSE results. We note that HWA is slightly more conservative than BSE, resulting in lower probabilities of a miss. FSS generated good segmentations, except for one Alzheimer's subject (with a Jaccard similarity coefficient of $J=0.56$ ). WAT segmentations were conservative, always including lots of nonbrain dark tissue (CSF, bone...). On this data set, BET did not perform well: Brain segmentations were accurate around the upper cerebral cortex, but often included large neck regions of nonbrain tissue, leading to lower Jaccard similarity coefficients. Table 1 and Fig. 10 summarize the results.

\section{Second data set}

The second data set was of lower quality than the first one. HWA performed the best. On this data set, BET performed significantly better but still included some nonbrain tissue in the neck region. BSE did not give satisfying results. WAT performed well but failed on one subject. Our method was able to recover from the failing watershed segmentation. FSS was the only method that clearly performed more poorly on images of demented adults than others [with $J=0.633$ (0.047) compared to $J=0.728(0.022)$ for nondemented adults]. Results can be found in Table 2 and Fig. 11.

\section{Discussion and risk evaluation}

On these two data sets, HWA outperformed other methods, resulting in segmentations with better Jaccard similarity coefficients. No method seemed to be sensitive to a specific group of individuals, except FSS that did not give satisfactory results over images of demented adults. We note that although FSS achieved good results, it consistently shaved off parts of the cortex. On the other hand, BET proved to be more conservative than other

Table 1

Mean (standard error) coefficients for Jaccard similarity, probability of a miss and probability of false detection for the first data set

\begin{tabular}{llll}
\hline Algorithm & $J$ & $p_{\mathrm{f}}$ & $p_{\mathrm{m}}$ \\
\hline FSS & $0.718(0.046)$ & $0.242(0.016)$ & $0.039(0.040)$ \\
BET & $0.483(0.171)$ & $0.510(0.176)$ & $0.007(0.007)$ \\
BSE & $0.751(0.104)$ & $0.181(0.120)$ & $0.070(0.024)$ \\
WAT & $0.681(0.039)$ & $0.315(0.040)$ & $0(0)$ \\
HWA & $0.885(0.023)$ & $0.010(0.026)$ & $0.014(0.007)$ \\
\hline
\end{tabular}

Table 2

Mean (standard error) coefficients for Jaccard similarity, probability of a miss and probability of false detection for the second data set

\begin{tabular}{llll}
\hline Algorithm & $J$ & $p_{\mathrm{f}}$ & $p_{\mathrm{m}}$ \\
\hline FSS & $0.702(0.052)$ & $0.261(0.040)$ & $0.036(0.041)$ \\
BET & $0.570(0.156)$ & $0.428(0.155)$ & $0.001(0.003)$ \\
BSE & $0.541(0.128)$ & $0.428(0.193)$ & $0.030(0.026)$ \\
WAT. & $0.651(0.086)$ & $0.320(0.052)$ & $0.026(0.118)$ \\
HWA & $0.858(0.029)$ & $0.113(0.047)$ & $0.003(0.017)$ \\
\hline
\end{tabular}

techniques (low $p_{\mathrm{m}}$ ), but often included lots of nonbrain tissue in the neck area, leading to large probability of false detection. BSE performed really well on the first data set, but proved to have difficulties coping with the second data set of lower quality. Finally, we note that WAT is associated with a zero probability of a miss for the first data set.

In our view, it is significantly more important to avoid removing brain than to remove all nonbrain structures. For this reason, we introduced an error function $c \mapsto E(c)$ defined in "Risk evaluation" section. Fig. 12 reports the two error functions for both data sets. Our method minimized both risks for $1 \leq c \leq 10$.

Finally, we provide some skull stripping results in Fig. 13. In our experience, the proposed algorithm is robust to noise, artifacts and bias fields. Studies of other groups (FennemaNotestine et al., submitted for publication) have shown that our method outperformed several others, proved to be more sensitive and robust, and most successfully retained brain tissue even within the difficult $\mathrm{AD}$ group. We note that our method has been successfully used in several different studies, requiring automated brain segmentations (Fischl et al., 2002, 2004; Salat et al., in press).

\section{Conclusion}

Our goal, when implementing this new skull-stripping algorithm, was to develop an automated algorithm able to successfully segment the whole brain, without any user intervention. This new segmentation process, based on a hybrid approach, which combines watershed algorithms and deformable surface models, offers the user both the robustness of watershed algorithms and the accuracy of deformable surface models. Surface-based methods, which easily incorporate geometric information, do not have access to interior intensity information, whereas watershed methods ignore geometric information. Our Hybrid method, combining both approaches, achieves better results than either one individually. Specifically, we extend the watershed approach proposed by Hahn and Peitgen in (2000) and improve the robustness of the algorithm by introducing a postwatershed analysis that validates and eventually corrects the segmentation. Template-based approaches, which incorporate shape information into the segmentation process and model the brain by a smoothed deformed surface, have been successfully used to extract the brain from MR images (Dale et al., 1999; Smith). However, some parts of the brain are difficult to correctly extract and are subject to recurrent errors. In this work, we propose a new approach that incorporates an atlasbased term, which preserves the shape of the surface during the deformation. The atlas, compiled from a set of successfully 

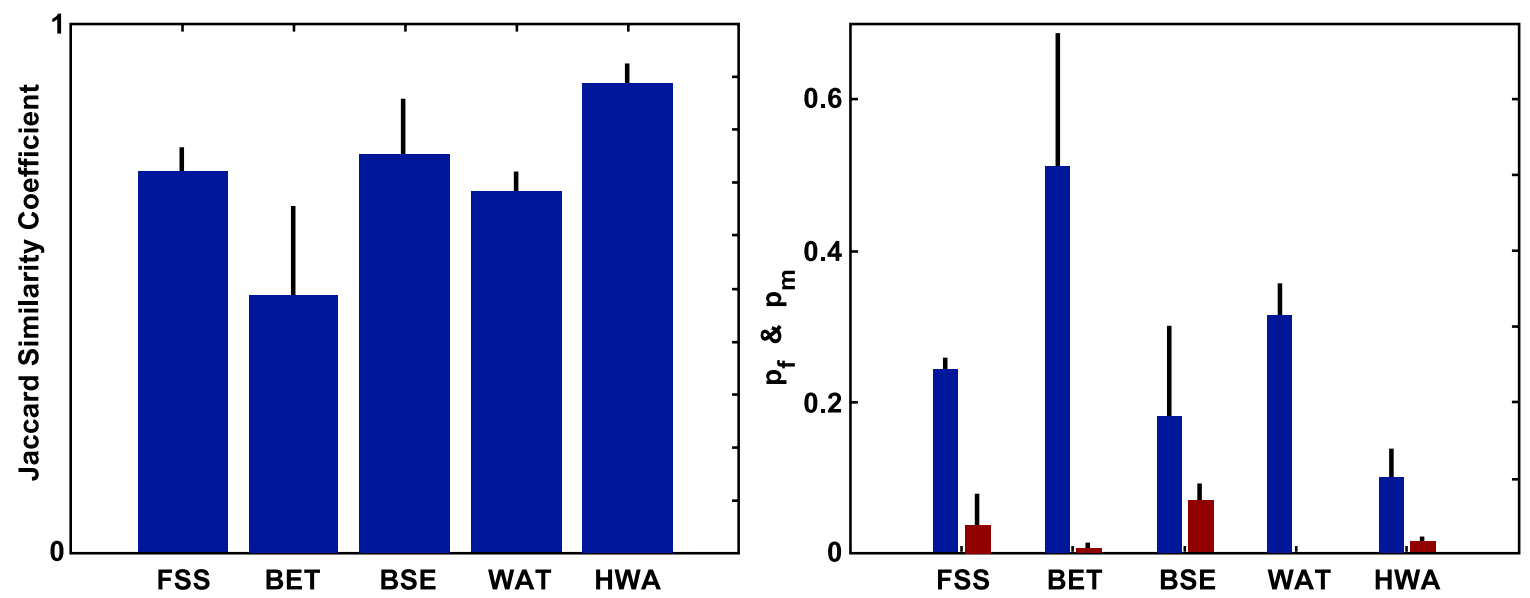

Fig. 10. Bar chart of the mean and standard errors for the Jaccard Similarity Coefficient (left), the probability of false detection and probability of a miss (right): first data set.
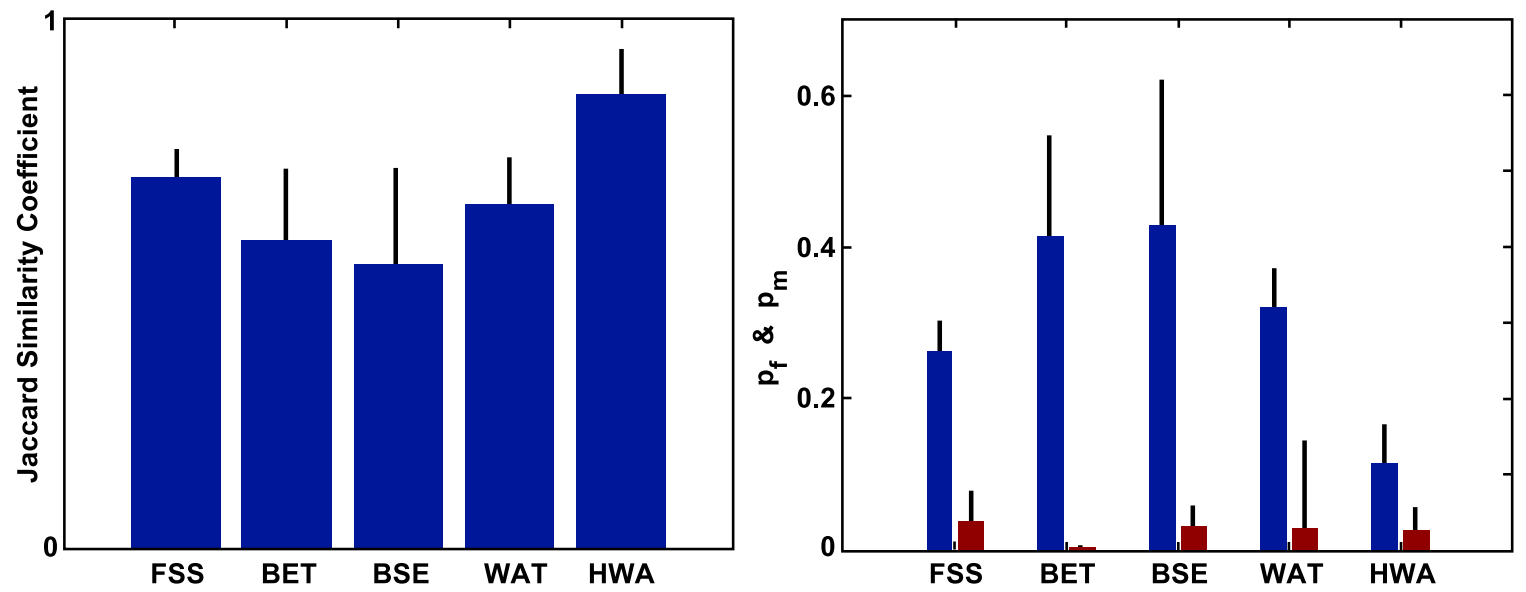

Fig. 11. Bar chart of the mean and standard errors for the Jaccard similarity coefficient (left) and the probability of false detection and probability of a miss (right): second data set.
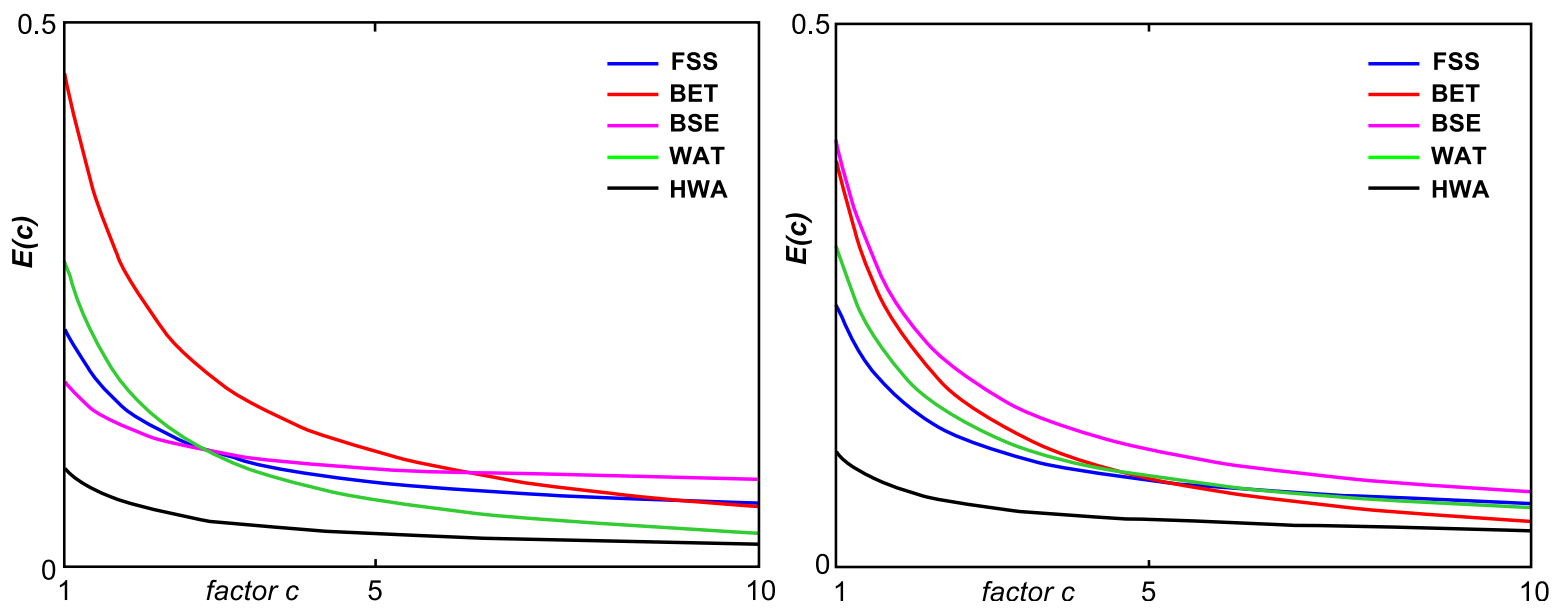

Fig. 12. Evaluation of the risk: $c \mapsto E(c)$. 


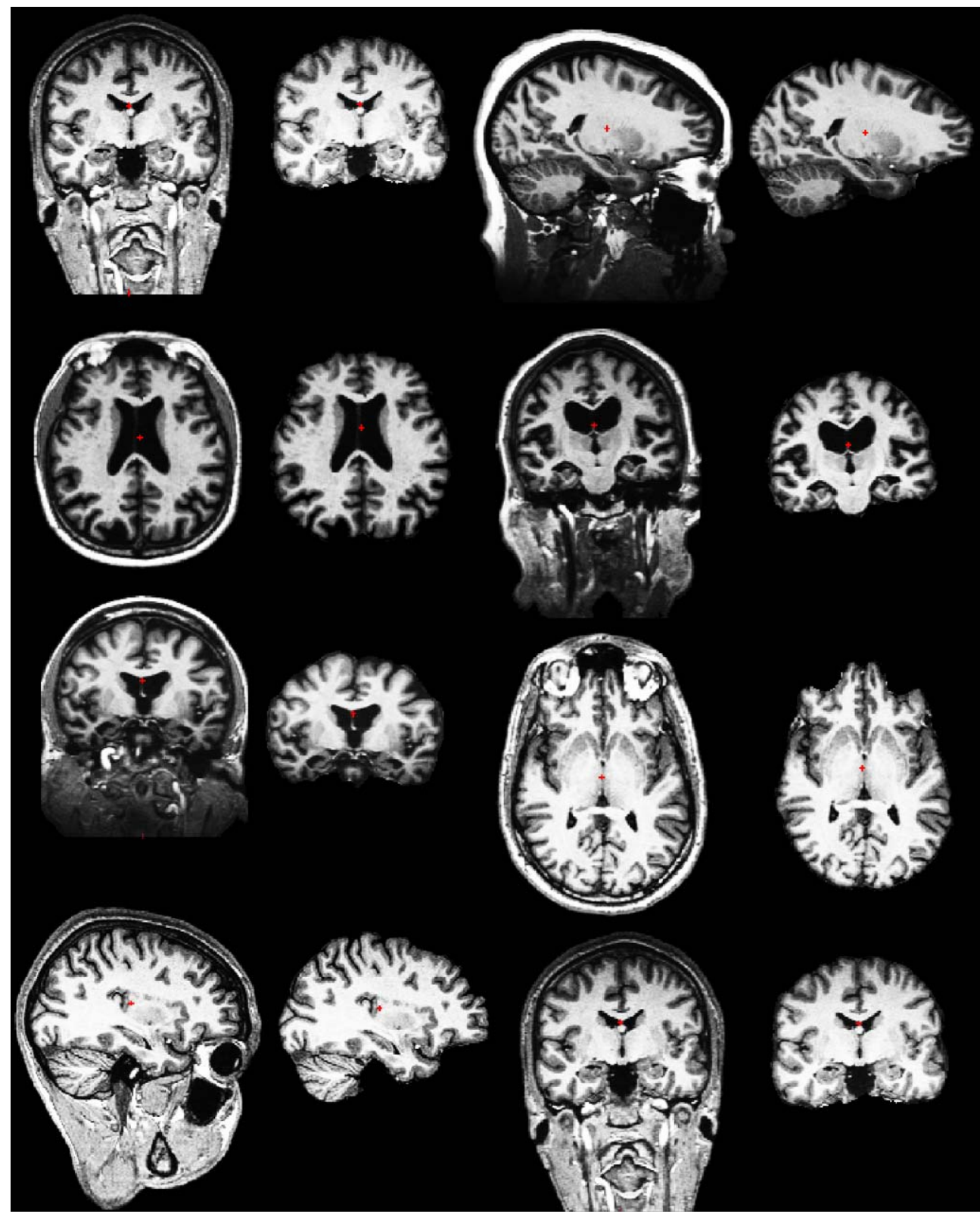

Fig. 13. Results of the proposed method.

segmented brains, is used to verify the shape of the brain surface and to correct it if needed.

The validation of the method has been achieved through the comparison of our results with ones provided by other methods (Dale et al., 1999; Hahn and Peitgen, 2000; Smith), and several manually segmented brains. Our method performed best overall, leading to few misclassified voxels. The main advantages are its robustness and its accuracy, as the final surface accurately fits the outer boundary of the pial surface of the cortex. The algorithm is fast ( $\approx 5$ min on a $1-\mathrm{GHz}$ Pentium III running Linux), and does not require any preprocessing of the image such as spatial or intensity normalization. Other group studies (Fennema-Notestine et al., submitted for publication) have shown that our method outperformed several others. We note that this method has been successfully used in many studies (Fischl et al., 2002, 2004; Salat et al., in press).
This skull-stripping technique is part of the cortical surface reconstruction and flattening software Freesurfer, associated with Dale et al. (1999) and Fischl et al., (1999a,b, 2001).

\section{Acknowledgments}

This work was supported in part by the National Center for Research Resources (P41-RR14075, R01 RR16594-01A1), the NCRR BIRN Morphometric Project BIRN002), as well as the Mental Illness and Neuroscience Discovery (MIND) Institute. The authors would like to thank Dr. Olivier Faugeras (Project RobotvisI.N.R.I.A) for his help and contribution to this research, Randy Buckner and the Washington University Alzheimer's Disease Research Center for providing data and the anonymous reviewers for their positive remarks, which greatly improved the paper. 
Appendix A. To clarify the presentation, certain explanations, which can be ignored in a first lecture of the paper, are described in this section.

\section{A.1. Preprocessing}

Computation of estimates of the CSF intensity, COG and brain radius: Following the work of Stephen Smith in BET (Smith), we first compute estimates of a set of parameters required for subsequent processing. These include an upper bound on the intensity of the cerebrospinal fluid $\left(\mathrm{CSF}_{\mathrm{MAX}}\right)$, the coordinates of the centroid of the brain (COG), and an estimate of the average radius of the brain. We proceed as follows: Ignoring very bright and dark voxels, which are defined as voxels whose intensity lies above $98 \%$ of the cumulative histogram of the image and below $2 \%$, respectively, the CSF threshold is set to lie $10 \%$ of the way between the minimum intensity and the maximum intensity in the image. This initial estimate is used to roughly distinguish between brain and nonbrain tissues (cerebrospinal fluid, skull...). Then, the voxels that are classified as brain are used to estimate the centroid of the brain: every voxel, whose intensity is above the previously calculated $\mathrm{CSF}_{\mathrm{MAX}}$ threshold, is used in a standard sum of position weighted by their intensity. Finally, a rough estimate of the brain/head radius is calculated.

Estimation of the white matter parameters: To determine the white matter parameters, we construct a curve using the fact that, in T1-weighted MRI, the white matter can be identified as a uniform region localized in the center of the head, with a quasi-constant intensity. The white matter parameters are computed from a cubic region $\mathrm{CR}$ centered on the centroid of the brain volume. The length of each edge of the cube is half the previously estimated brain radius (see Fig. 2). Within this region, the number of white matter voxels should be larger than the number of non-white-matter voxels, and the corresponding average variance should be smaller. Therefore, we build a histogram $f$ indexed by the intensity $i f: i \mapsto[n(i)] /[v(i)]$, where $n(i)$ is the number of voxels having an intensity equal to $i$, and $v(i)$ is the average variance of these voxels. The curve $f$ should have a sharp maximum for the white-matter-intensity values, leading to histogram estimates that are not corrupted by presence of other tissue classes. We proceed as follows.

For each voxel $k$ within the cube, a 27-neighbor mean $I_{k}$ and variance $v_{k}$ is computed. For each intensity $i$ encountered in the region of interest, we calculate the number of voxels $k$ having a mean intensity $i: n(i)=\sum_{\text {voxels } k \in \mathrm{CR}} \delta\left(I_{k}, i\right)$, where $\delta$ is the classic delta function: $\delta\left(I_{k}, i\right)=1$ if $I_{k}=i, 0$ otherwise. The average variance of these voxels is then equal to: $v(i)=\frac{1}{n(i)} \sum_{\text {voxels } k \in \mathrm{CR}} \delta\left(I_{k}, i\right) v_{k}$. The function $f$, indexed by the intensity $i$, is used to calculate class statistics of white matter. We first localize the main lobe of the function $f$, defined as the region where $f$ reaches its maximum $f_{\max }$ (over a 5-point window), and for which $f(i)$ is greater than $f_{\max } / 3$; the bounds of the main lobe region are used to define $\mathrm{WM}_{\mathrm{MIN}}$ and $\mathrm{WM}_{\mathrm{MAX}}$. Fig. 5 provides an example. Then, within this region, we calculate the average variance $\sigma^{\mathrm{WM}}$ of the white matter, using all voxels with intensity within the main lobe of the function $f$ (Fig. 14).

\section{A.2. The watershed algorithm}

The watershed algorithm proceeds in two steps. We first apply on the image a watershed transform as the one proposed by Hahn and Peitgen in (2000). Then, we assess the validity of the watershed segmentation and retrospectively correct it if necessary.

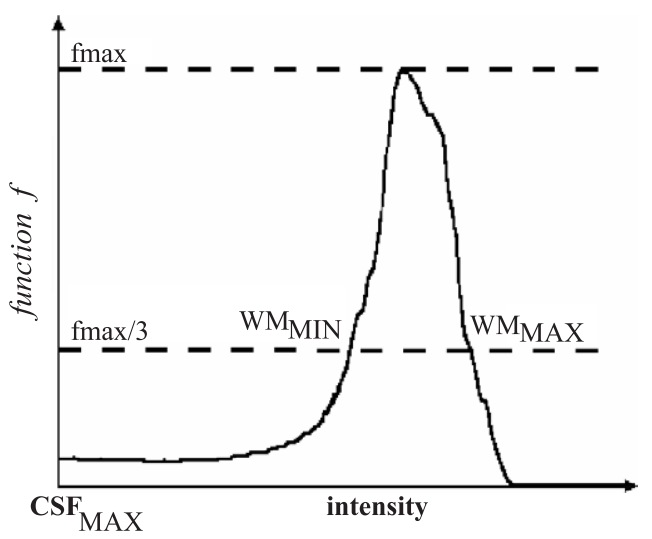

Fig. 14. An example of the function $f$ used to compute the intensity characteristics of white matter.

Watershed transform: The first step of the watershed algorithm is the sorting of all voxels (of the gray level inverted image) according to their intensity. Then, we process each voxel in ascending order: If the voxel does not have any already processed neighbors in its three-dimensional 6-neighborhood (i.e., voxels of same or less intensity), a new basin is formed. This voxel represents a local intensity minimum. Otherwise, we merge the voxel with the deepest neighboring basin, i.e., the basin with the darkest bottom voxel: "Voxel-basin merging". If two or more neighbors have already been processed belonging to different basins, these are tested for "basin-basin merging": All the neighboring basins whose depth relative to the current voxel intensity is less than or equal to the preflooding height $h_{\mathrm{pf}}$ will be merged with the same basin as the voxel itself, i.e., the deepest neighboring basin (Fig. 15).

After the transform with an appropriate preflooding height, one basin should exist that represents the whole brain, and will include the previously identified white-matter voxel in "The watershed algorithm" section above.

The complexity of the modified watershed transform is linear in the number of voxels $N$ (usually on the order of $N=256 \times$ $256 \times 256)$ for the voxel processing, and in the worst case proportional to $N \log N$ for the sorting. However, taking into account the fact that the intensity of each voxel intensity can be represented with a single byte (range from 0 to 255), we can accomplish the sorting in $N$ operations, resulting in a watershed transform whose computational complexity is linear in the number of voxels.

Post-watershed correction: The resulting image is often inaccurate and nonsmooth, with extracerebral tissues and CSF frequently remaining. In addition, some artifacts in the original image, such as susceptibility artifacts, may cause an incorrect segmentation. The brain may be split in two or more basins, and the resulting segmentation may give an inaccurate result. For these reasons, we automatically check the reasonableness of the initial watershed segmentation and correct it where possible.

We first determine whether the size of the main basin seems to be correct. We use the previously calculated brain radius to estimate the volume of the brain, assuming spherical geometry. If the size of the main basin is significantly smaller than the estimated volume (four times smaller), we assume that the watershed segmentation failed and needs to be corrected. This is accom- 

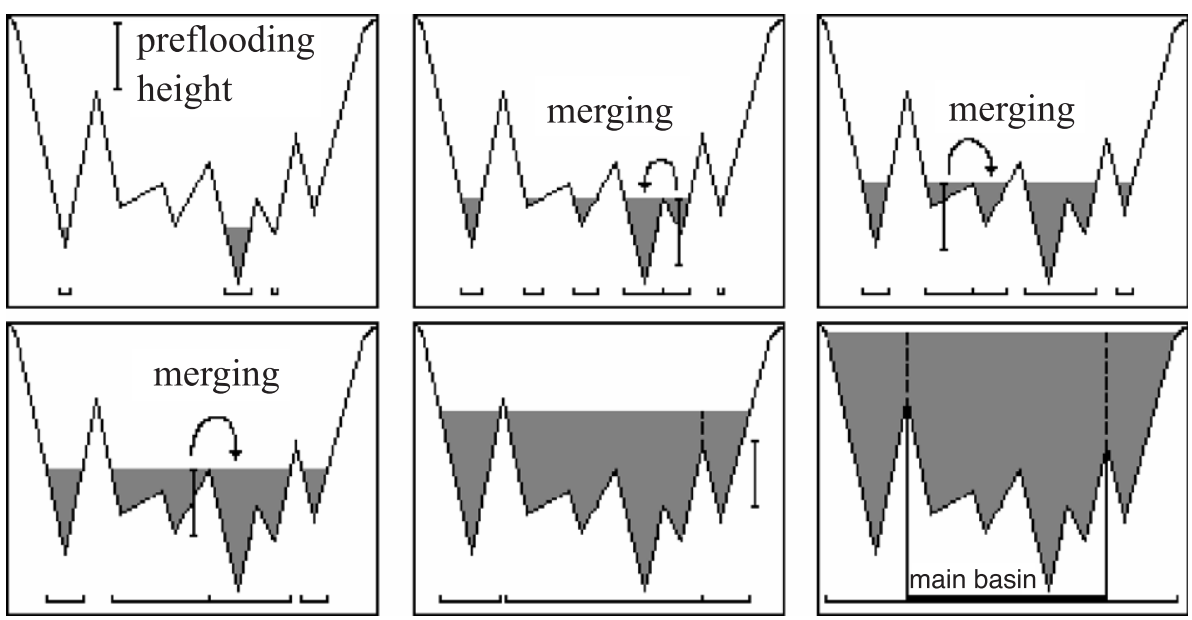

Fig. 15. A simple illustration of the merging process. A basin is merged into a deeper basin, if and only if its depth relative to the current voxel intensity is less than or equal to the preflooding height.

plished by merging basins into the main basin representing the initial segmentation. The basin we merge is one that is adjacent to the main basin, localized in the white matter, and whose volume would result in the segmentation being the closest to the estimated brain volume. In the cases in which the watershed segmentation fails, few basins are found and the chosen basin is often the biggest. In our experience, this post-watershed basin merging frequently results in a correct segmentation.

Nevertheless, some artifacts segment the brain in many equally sized basins. In this case, further corrections are required. To correct this type of failure, we merge all the ambiguous basins with the main one. An ambiguous basin is defined as a basin that contains a significant volume of white matter at its common border with the already segmented basin. A voxel is said to be ambiguous if it is a neighbor of the main basin, if its intensity is greater than $\mathrm{WM}_{\mathrm{MIN}}$ and smaller than $\mathrm{WM}_{\mathrm{MAX}}$, with a variance smaller than $\sigma_{\mathrm{WM}}$. If the number of ambiguous voxels is greater than a specific threshold, then we label this basin as "ambiguous", and merge it to the brain basin. The empirical threshold, $t_{\mathrm{m}}$, we are using is equal to the cube root of the considered basin size: if the number of ambiguous voxels in an uncertain basin is greater than the cube root of its size, then we merge it to the main one. We found that the use of a threshold depending on the size of the targeted basin leads to better results: it avoids merging some large basins with a comparatively small number of ambiguous voxels. We iterate this process until no new basin is merged with the main one. Usually, even with an oversegmentation of the brain basin into many different smaller basins, this post-watershed analysis merges most of them in a few passes:

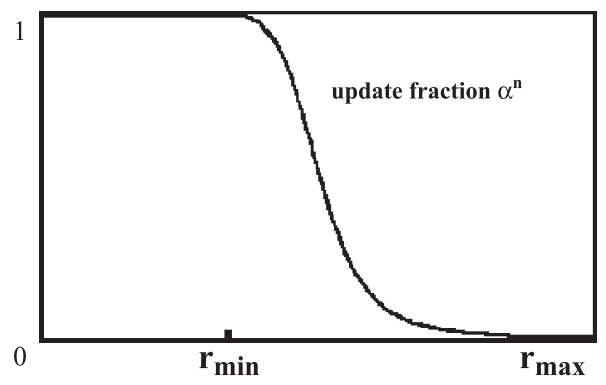

Fig. 16. The smoothness update fraction. typically 2 or 3 . In our experience, most watershed segmentations are correct and do not require post-watershed corrections.

\section{A.3. Deformable surface algorithm}

Computation of the curvature reducing force, $\mathbf{F}_{\mathbf{S}}$ : The curvature reducing force $\mathbf{F}_{\mathrm{S}}$, which enforces a smoothness constraint on the deformed template, is based on the work of Smith.

At each iteration $t$, for each vertex $k$, the mean position $\left(\overline{\boldsymbol{x}}_{k}^{t}\right.$ of all its neighboring vertices is calculated to form a difference vector $\boldsymbol{s}_{k}=\boldsymbol{x}_{k}^{t}-\overline{\boldsymbol{x}}_{k}^{t}$, which is decomposed into orthogonal components, normal and tangential to the surface: $\boldsymbol{s}_{k}=\boldsymbol{s}_{k}^{n}+\boldsymbol{s}_{k}^{t}$. The smoothness force used in Dale et al. (1999) is given by: $\mathbf{F}_{S}$ $\left(\boldsymbol{x}_{k}^{t}\right)=\alpha^{n} s_{k}^{n}+\alpha^{t} s_{k}^{t}$, with $\alpha^{n}$ and $\alpha^{t}$ as two constants (usually set to 0.5 ). This approach can be significantly improved by making $\alpha^{n}$ a nonlinear function of $\boldsymbol{s}_{k}^{n}$. In this way, small departures from planarity are not penalized, while large ones are disallowed. More specifically, we proceed as follows. We estimate the local radius of curvature $r_{k}$ at vertex $k: r_{k}=\left(\bar{d}_{k}^{2}\right) /\left(2\left|s_{k}{ }^{n}\right|\right.$, where $\bar{d}_{k}$ is the average distance from vertex $k$ to its neighboring vertices. Then, the update fraction is set to a sigmoid function of $r_{\mathrm{k}}$ :

$\alpha^{n}=\frac{1}{2}\left[1+\tanh \left(B^{*}\left(\frac{1}{r_{k}}-A\right)\right)\right]$,

with $A$ and $B$ equal to: $A=\left[1 / r_{\min }\right]+\left[1 / r_{\max }\right]$ and $B=6\left[\left(1 / r_{\min }\right)-\right.$ $\left.\left(1 / r_{\max }\right)\right]$. The two parameters $r_{\min }$ and $r_{\max }$ reflect prior hypotheses

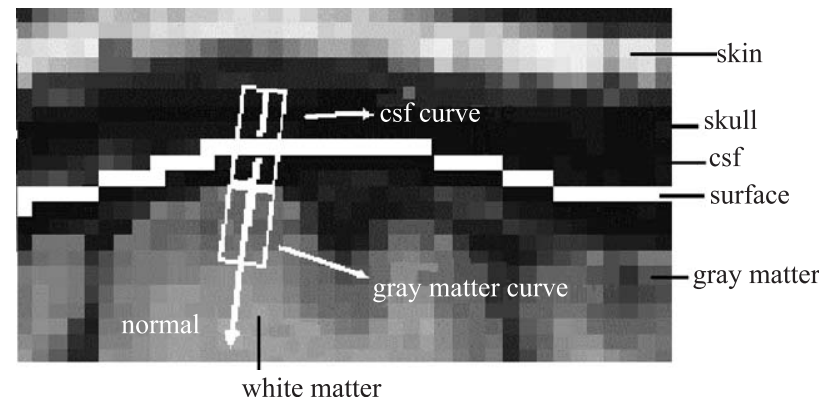

Fig. 17. Construction of the CSF and GM curves. 


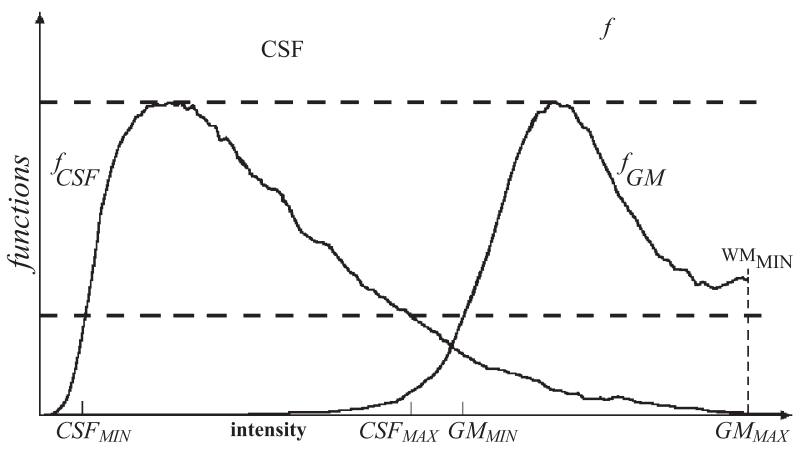

Fig. 18. The CSF and GM curves.

about the allowable curvature of the brain/nonbrain boundary and are set to $r_{\min }=3.33$ and $r_{\max }=10$. The sigmoid function (see Fig. 16) penalizes high local mean curvatures of the surface, where $1 /$ $r_{\text {min }}$ is assumed to be an upper bound to the expected local curvature. Conversely, regions with low local mean curvature (of the order of $1 / r_{\max }$ or smaller) are little regularized by the curvature reducing force. Future work will incorporate atlas-based information to locally set these two parameters.

Computation of global parameter: After the coarse initialization described in "Initialization of the deformable model" section, we need to compute a few parameters regarding the intensity values at the brain/nonbrain boundary, such as the mean intensity and variance of CSF and gray matter. We compute these parameters by examining the intensity values along the surface normals, extending from a few millimeters inward to a few millimeters outward from the previously calculated surface. Along these line segments, similarly to the estimation of the white matter parameters in Appendix A.1, we construct two global cumulative histograms, which reach their maxima around the mean CSF and GM intensities (Fig. 17).

Construction of the CSF curve and extraction of some CSF parameters: As part of the watershed processing, we have already determined an upper bound $\mathrm{CSF}_{\mathrm{MAX}}$ for the value for the CSF intensity (see "Preprocessing" section). This value now needs to be refined. Our first estimation corresponds to a simple threshold that has been set to roughly distinguish between brain and nonbrain tissues, based on the cumulative histogram of the image. It did not directly use CSF intensity information. However, the surface that is a result of the coarse template deformation, described in "Initialization of the deformable model" section, mostly passes through "dark" regions in the T1-weighted image, corresponding to CSF or skull. For each vertex of the surface, looking 2-mm outward and 2-mm inward among 5 voxels, we find the voxel with the smallest intensity, and keep it for the construction of the CSF curve if its intensity $I_{k}$ is less than $3 \mathrm{CSF}_{\mathrm{MAX}}$ (we ignore bright areas where the surface passes, such as the eye sockets):

$f_{\mathrm{CSF}}(i)=\sum_{\text {all kept voxels } k} \delta\left(I_{k}, i\right)$.

Following the same approach as for the estimation of the white matter parameters, we localize the main lobe of the function $f$ : we find the maximum of the curve, i.e., the new CSF intensity value $\mu^{\mathrm{CSF}}$, and identify two inferior and superior limits of the main lobe: $\mathrm{CSF}_{\text {MIN }}$ and $\mathrm{CSF}_{\text {MAX }} \sigma .{ }^{\mathrm{GM}}$. Fig. 18 shows an example of the CSF curve. Then, we estimate the variance of the CSF: Every voxel with intensity within the main lobe is used to compute a maximum likelihood estimate of the variance $\sigma^{\mathrm{CSF}}$.
Construction of the gray matter curve: The gray matter curve is obtained looking 20-mm along the inward normal. Along the normal of each vertex of the tessellation, progressing iteratively mm-by-mm inward, we search for a uniform white matter area, that we defined as a $3 * 3 * 3$-voxel region, in which the average intensity falls between $\mathrm{WM}_{\mathrm{MIN}}$ and $\mathrm{WM}_{\mathrm{MAX}}$, with a variance is smaller than $\sigma^{\mathrm{WM}}$. As soon as we succeed in localizing this area, meaning that we have identified a uniform white-matter region along the inward normal, we use the voxels located along the normal, between this area and the tessellated surface, to increment a cumulative histogram, as we previously did for the CSF curve. These voxels, bounded by white-matter voxels and CSF ones, are expected to represent gray matter. Then, we extract the gray matter parameters, such as the GM mean intensity $\mu^{\mathrm{GM}}$ and variance $\sigma^{\mathrm{GM}}$ (see Fig. 18). We note that some WM regions might be ignored in certain parts of the brain due to intensity inhomogeneities or noise differences, leading to biased estimates of the brain parameters. However, the goal of this coarse estimation is to generate a unique threshold able to roughly distinguish between GM and CSF. The contrast between gray matter and CSF is usually sufficiently large that using unique predefined global transition intensity should suffice to distinguish the two tissues. Besides, the number of iterations in the subsequent iterative surface deformation is restricted to a few iterations, preventing large departures from the coarse initialization.

\section{A.4. Table of parameters}

\begin{tabular}{|c|c|c|c|}
\hline Parameters & Defined in... & Estimated? & Value \\
\hline $\mathrm{CSF}_{\text {MAX }}$ & $\begin{array}{l}\text { "Preprocessing" } \\
\text { section; } \\
\text { Appendix A.1 }\end{array}$ & Yes & - \\
\hline Brain radius, $\mathrm{BR}$ & $\begin{array}{l}\text { "Preprocessing" } \\
\text { section; } \\
\text { Appendix A.1 }\end{array}$ & Yes & - \\
\hline $\begin{array}{l}\text { Centroid of } \\
\text { the brain, COG }\end{array}$ & $\begin{array}{l}\text { "Preprocessing" } \\
\text { section; Appendix A.1 }\end{array}$ & Yes & - \\
\hline $\begin{array}{l}\text { White matter parameters, } \\
\mathrm{WM}_{\mathrm{MIN}} \text {, } \\
\mathrm{WM}_{\mathrm{MAX}} \text { and } \sigma_{\mathrm{WM}}\end{array}$ & $\begin{array}{l}\text { "Preprocessing" } \\
\text { section; Appendix A.1 }\end{array}$ & Yes & - \\
\hline Preflooding height, $h_{\mathrm{pf}}$ & $\begin{array}{l}\text { "The watershed } \\
\text { algorithm" } \\
\text { section; Appendix A.2 }\end{array}$ & No & $\begin{array}{l}25 \% \\
\text { of } I_{\mathrm{MAX}}\end{array}$ \\
\hline $\begin{array}{l}\text { Post-watershed } \\
\text { threshold, } t_{\mathrm{m}}\end{array}$ & Appendix A.2 & Yes & $\sqrt[3]{ }$ \\
\hline $\begin{array}{l}\text { Global brain parameters, } \\
\mu^{\mathrm{CSF}}, \mu^{\mathrm{GM}}, \\
\sigma^{\mathrm{CSF}} \text { and } \sigma^{\mathrm{GM}}\end{array}$ & $\begin{array}{l}\text { "Initialization of the } \\
\text { deformable model" } \\
\text { section; } \\
\text { Appendix A.3 }\end{array}$ & Yes & - \\
\hline$r_{\min }$ & $\begin{array}{l}\text { "Active contour } \\
\text { formulation: } \\
\text { the parametric } \\
\text { deformable model" } \\
\text { section; } \\
\text { Appendix A.3 }\end{array}$ & No & 3.33 \\
\hline$r_{\max }$ & $\begin{array}{l}\text { "Active contour } \\
\text { formulation: } \\
\text { the parametric } \\
\text { deformable } \\
\text { model" section; } \\
\text { Appendix A.3 }\end{array}$ & No & 10 \\
\hline
\end{tabular}


Table (continued)

\begin{tabular}{|c|c|c|c|}
\hline Parameters & Defined in... & Estimated? & Value \\
\hline$\lambda_{\mathrm{D}}$ & $\begin{array}{l}\text { "Local parameters } \\
\text { estimation } \\
\text { and final deformation" } \\
\text { section }\end{array}$ & No & 0.25 \\
\hline$\lambda_{\mathrm{C}}$ & $\begin{array}{l}\text { "Local parameters } \\
\text { estimation } \\
\text { and final deformation" } \\
\text { section }\end{array}$ & No & 0.025 \\
\hline $\begin{array}{l}\text { Convergence } \\
\text { threshold }\end{array}$ & $\begin{array}{l}\text { "Local parameters } \\
\text { estimation } \\
\text { and final deformation" } \\
\text { section }\end{array}$ & No & $0.5 \mathrm{~mm}$ \\
\hline
\end{tabular}

\section{References}

Atkins, M.S., Mackiewich, B.T., 1998. Fully automatic segmentation of the brain in MRI. IEEE Trans. Med. Imaging 17 (1), 98-107.

Baillard, C., Hellier, P., Barillot, C., 2001. Segmentation of brain 3D MR images using level sets and dense registration. Med. Image Anal. 5, 185-194.

Buckner, R.L., Snyder, A.Z., Sanders, A.L., Raichle, M.E., Morris, J.C., 2000. Functional brain imaging of young, nondemented, and demented older adults. J. Cogn. Neurosci. 12 (Suppl. 2), 24-34.

Cox, R.W., 1996. AFNI: software for analysis and visualization of functional magnetic resonance neuroimages. Comput. Biomed. Res. 29 (3), $162-173$.

Dale, A.M., Sereno, M.I., 1993. Improved localization of cortical activity by combining EEG and MEG with MRI cortical surface reconstruction: a linear approach. J. Cogn. Neurosci. 5 (2), 162-176.

Dale, A.M., Fischl, B., Sereno, M.I., 1999. Cortical surface-based analysis: I. Segmentation and surface reconstruction. NeuroImage 9 (2), 179-194.

Dawant, B.M., et al., 1999. Automatic 3-D segmentation of internal structures of the head in mr images using a combination of similarity and freeform transformations: Part I. Methodology and validation on normal subjects. IEEE Trans. Med. Imaging 18 (10), 909-916.

Faugeras, O., et al., 1999. The Inverse EEG and MEG Problems: The Adjoint State Approach I: The Continuous Case. Rapport de Recherche INRIA.

Fennema-Notestine, C., et al., submitted for publication. Quantitative evaluation of automated skull-stripping methods applied to contemporary and legacy images: effects of diagnosis, bias correction, and slice location. Hum. Brain Mapp.

Fischl, B., Dale, A.M., 2000. Measuring the thickness of the human cerebral cortex using magnetic resonance images. Proc. Natl. Acad. Sci. 97, $11044-11049$.

Fischl, B., Sereno, M.I., Dale, A.M., 1999a. Cortical surface-based analysis: II. Inflation, flattening, and a surface-based coordinate system. NeuroImage 9 (2), 195-207 (Feb.).

Fischl, B., Sereno, M.I., Tootell, R.B.H., Dale, A.M., 1999b. High-resolution inter-subject averaging and a coordinate system for the cortical surface. Hum. Brain Mapp. 8 (4), 272-284.

Fischl, B., Liu, A., Dale, A.M., 2001. Automated manifold surgery: constructing geometrically accurate and topologically correct models of the human cerebral cortex. IEEE TMI 20 (1), 70-80.

Fischl, B., Salat, D.H., Albert, M., Dieterich, M., Haselgrove, C., van der Kouwe, A., Killiany, R., Kennedy, D., Montillo, A., Makris, N., Rosen, B., Dale, A.M., 2002. Whole brain segmentation: automated labeling of neuroanatomical structures in the human brain. Neuron 33 (3), 341-355 (Jan. 31).

Fischl, B., van der Kouwe, A., Destrieux, C., Halgren, E., Segonne, F.,
Salat, D.H., Busa, E., Seidman, L.J., Goldstein, J., Kennedy, D., Caviness, V., Makris, N., Rosen, B., Dale, A.M., 2004. Automatically parcellating the human cerebral cortex. Cereb. Cortex 14 (1), 11-22 (Jan.).

Goldenberg, R., et al., 2001. Cortex segmentation-A fast variational geometric approach. IEEE Workshop on Variational and Level Set Methods in Computer Vision, Vancouver, Canada. July.

Hahn, H.K., Peitgen, H.-O., 2000. The Skull Stripping Problem in MRI Solved by a Single 3D Watershed Transform. MICCAI 2000, Pittsburgh, PA, USA, pp. 134-143.

Kapur, T., Grimson, W.E.L., Kikinis, R., 1995. Segmentation of Brain Tissue from MR images. AITR-1566. June.

Kass, M., Witkin, A., Terzopoulos, D., 1988. Snakes: active contour models. Int. J. Omp. Vis. 1, 321-331.

Lemieux, L., et al., 1999. Fast, accurate, and reproducible automatic segmentation of the brain in T1-weithed volume MRI data. Magn. Reson. Med. 42 (1), 127-135 (Jul.)

Logan, J.M., Sanders, A.L., Snyder, A.Z., Morris, J.C., Buckner, R.L., 2002. Under-recruitment and nonselective recruitment: dissociable neural mechanisms associated with aging. Neuron 33, 827-840.

MacDonald, D., et al., 2000. Automated 3-D extraction of inner and outer surfaces of cerebral cortex from MRI. NeuroImage 12, 340-356.

Meegama, R.G.N., et al., 2001. Fully automated peeling technique for MR head scans. 3rd IEEE International Conference on Information, Communications and Signal Processing (ICICS'01), Singapore.

Pham, D.L., Prince, J.L., 1999. An adaptive fuzzy segmentation algorithm for three-dimensional magnetic resonance images. Presented at XVIth Conference on Information Processing in Medical Imaging (IPMI99), June 28-July 2.

Rajapakse, J.C., 1997. Statistical approach to segmentation of single-channel cerebral MR images. IEEE Trans. Med. Imaging 16 (2), 176-186 (Apr.).

Rehm, K., et al., 1999. Semi-automated stripping of T1 MRI volumes: I. Consensus of intensity-and edge-based methods. NeuroImage 9 (6, Part 2 of 2), 86 .

Salat, D.H., et al., in press. Thinning of the cerebral cortex. Cereb. Cortex.

Shattuck, D.W., Leahy, R.M., in press. BrainSuite: an automated cortical surface identification tool [invited article]. Med. Image Anal.

Shattuck, D.W., Sandor-Leahy, S.R., Shaper, K.A., Rottenberg, D.A., Leahy, R.M., 2001. Magnetic resonance image tissue classification using a partial volume model. NeuroImage 13 (5), 856-876.

Smith, S., 2002. Fast robust automated brain extraction. Human Brain Mapping 17, 143-155

Thompson, P.M., Toga, A.W., 1996. A surface-based technique for warping 3-dimensional images of the brain. IEEE Trans. Med. Imaging $15,1-16$.

Thompson, P.M., MacDonald, D., et al., 1996. Detection and mapping of abnormal brain structure with a probabilistic atlas of cortical surfaces. J. Comput. Assist. Tomogr. 21 (4), 567-581.

Van Essen, D.C., Drury, H.A., 1997. Structure and functional analyses of human cerebral cortex using a surface-based atlas. J. Neurosci. 17 (18), 7079-7102.

Ward, B.D., 1999. Intracranial segmentation. Biophysics Research Institute, Medical College of Wisconsin, Milwaukee, WI.

Wells, W.M., Kikinis, R., Grimson, W.E.L., Jolesz, F., 1996. Adaptive segmentation of MRI data. IEEE Transactions on Medical Imaging $15,429-442$.

Xu, C., et al., 1999. Reconstruction of the human cortical surface from MR images. IEEE Trans. Med. Imaging 18 (6) (June).

Zeng, X., et al., 1999. Segmentation and measurement of the cortex from 3D MR images using coupled surfaces propagation. IEEE Trans. Med. Imaging 18 (10), 100-111.

Zhang, Y., Brady, M., Smith, S., 2001. Segmentation of brain MR images through a hidden Markov random field model and the expectationmaximization algorithm. IEEE Transactions on Medical Imaging 20, $45-57$. 